Revisitando la hipótesis de Frederic Engel: cómo las lomas determinan la distribución arqueológica del Precerámico en la costa sur del Perú

David G. Beresford-Jones, George Chauca Iparraguirre, Kevin J. Lane, Oliver Q. Whaley, Justin Moat, Alexander G. Pullen, Susana Arce Torres, Alfonso Orellana García, Lauren Cadwallader, Jorge Rodriguez, Delphine Joly, Charles A. I. French

Relaciones, 46(2), e014, julio-diciembre 2021 ISSN 1852-1479 | https://doi.org/10.24215/18521479e014 https://revistas.unlp.edu.ar/relaciones

ISSN 0325-2221 (versión impresa) Sociedad Argentina de Antropología (SAA)

Buenos Aires I Argentina

\title{
REVISITANDO LA HIPÓTESIS DE FREDERIC ENGEL: CÓMO LAS LOMAS DETERMINAN LA DISTRIBUCIÓN ARQUEOLÓGICA DEL PRECERÁMICO EN LA COSTA SUR DEL PERÚ
}

\author{
David G. Beresford-Jones *, George Chauca Iparraguirre**, Kevin J. Lane**, \\ Oliver Q. Whaley ${ }^{* * * * *}$, Justin Moat ${ }^{* * * * *}$, Alexander G. Pullen ${ }^{* * * * * *}$, \\ Susana Arce Torres ${ }^{* * * * * * *}$, Alfonso Orellana García ${ }^{* * * * * * * *}$, Lauren Cadwallader ${ }^{* * * * * * * *}$, \\ Jorge Rodriguez ${ }^{* * * * * * * *}$, Delphine Joly ${ }^{* * * * * * * * *}$ y Charles A. I. French ${ }^{* * * * * * * * *}$
}

Fecha de recepción: 28 de diciembre de 2020

Fecha de aceptación: 18 de junio de 2021

\footnotetext{
*Heinz Heinen Centre for Advanced Study, University of Bonn, Alemania, E-mail: david.beresfordjones@ gmail.com

${ }^{*}$ Universidad Nacional Mayor de San Marcos, Escuela Profesional de Arqueología, Lima, Perú. E-mail: georgechauca@yahoo.com

*** Consejo Nacional de Investigación de Ciencia y Tecnología - Instituto de las Culturas (IDECU), Universidad de Buenos Aires, Argentina. E-mail: kevin.lane@ cantab.net

**** Royal Botanical Gardens Kew, Richmond, Surrey, Reino Unido. E-mail: O.Whaley@kew.org

***** Royal Botanical Gardens Kew, Richmond, Surrey, Reino Unido. E-mail: J.Moat@kew.org

****** Pre-Construct Archaeology, The Granary, Rectory Farm, Pampisford, Reino Unido. E-mail: agp27@ hotmail.com

******** Museo Regional de Ica, Ministerio de Cultura, Ica, Perú. E-mail: susarceto@yahoo.com

******** Proyecto Kew Perú: Conservación, Restauración de Hábitats y Medios de Vida Útiles, Ica, Perú. E-mail: bio_aog@hotmail.com

******** Lauren Cadwallader, PLOS, Carlyle House, Carlyle Road, Cambridge, Reino Unido, E-mail: lcadwallader@plos.org

******** Universidad Nacional Mayor de San Marcos, Escuela Profesional de Arqueología, Lima, Perú. E-mail: j.rodriguez.mor@outlook.com

******** Department of Environment and Geography, Environment Building, University of York, Reino Unido. E-mail: delphine.joly@ york.ac.uk.

********* University of Cambridge, Department of Archaeology, Reino Unido. E-mail: caif2@ cam.ac.uk
} 
Relaciones de la Sociedad Argentina de Antropología 46 (2), julio-diciembre 2021: 383-413

\section{RESUMEN}

Frédéric Engel (1981:24), el pionero de los estudios del Precerámico en el Perú, argumentó que la distribución de los sitios arqueológicos durante el Holoceno temprano fue determinada por "la capacidad de las lomas" -oasis estacionales producidos por la humedad oceánica que están presentes a lo largo de la costa del Perú- una proposición que aún se encuentra en debate. Nosotros evaluaremos las ideas de Engel basándonos en datos de sitios arqueológicos del Precerámico Medio (hasta 4500 años cal AP) ubicados dentro y cerca de las lomas de la costa sur del Perú.

Palabras clave: Precerámico Medio - Arcaico - lomas - costa sur del Perú - cazadores-recolectores marinos

\section{REVISITING FREDERIC ENGEL'S HYPOTHESIS: HOW THE LOMAS DETERMINE THE DISTRIBUTION OF PRECERAMIC ARCHAEOLOGY ON PERU'S SOUTHERN COAST}

\section{ABSTRACT}

The pioneer of Preceramic archaeology in Peru, Frédéric Engel (1981: 24), argued that archaeological site distribution during the Early Holocene, was defined by the 'fog oasis situation' of lomas -the seasonal oases sustained by ocean fogs that flourish along the arid Pacific coast of Peru-a proposition that remains much debated.

Here we reassess Engel's ideas using archaeological findings from Middle Preceramic archaeological sites (to c. $4500 \mathrm{cal}$ BP) from alongside and within the lomas of the south coast of Peru.

Keywords: Middle Preceramic-Archaic-lomas-south coast Peru-marine hunter-gatherers

Hace casi 50 años desde que Engel (1973:271, nuestra traducción) escribió que "la importancia de las lomas con respecto a la geografía humana prehistórica, en su capacidad como áreas productoras de agua y alimentos, ha sido prácticamente ignorada". A pesar del tiempo transcurrido, aún se discute el rol que las lomas -"oasis nacido de las brumas", según Dollfus (1968)- jugaron en la larga historia de la ecología humana de la árida costa del Perú.

Frédéric Engel fue quizá el primer investigador de la prehistoria peruana en colocar en el centro de sus interpretaciones la interacción humana con los cambios ambientales del pasado. Por tanto, fue Engel un pionero de lo que Butzer (1982) definió más tarde como "la arqueología como la ecología humana". La enorme contribución de Engel a la arqueología peruana aún sigue siendo desconocida y subestimada (Velarde 2002-03; Benfer 2005), en parte porque su ambicioso proyecto de prospectar vastas áreas culminó -inevitablemente- en reportes breves y sin los detalles necesarios. Sin embargo, sus trabajos son transcendentales porque su equipo y él registraron por primera vez muchos de los sitios arqueológicos precerámicos más importantes del Perú, incluidos Paloma, El Paraiso, Asia Unidad 1, Quebrada Jaguay y, junto con su director de campo Bernandino Ojeda, quizá el sitio precerámico más emblemático del Perú, Caral. Asimismo, su trabajó coincidió con la primera fase de lo que fue la "revolución del radiocarbono", obteniendo los primeros fechados de varios sitios muy antiguos, mucho de los cuales son aún muy confiables (Benfer 2005; Sandweiss, comunicación personal). Con el tiempo, su influencia se extendió a través del trabajo de los numerosos arqueólogos distinguidos que habían comenzado sus carreras trabajando para Engel -entre ellos Henning Bischof, Claude Chauchat, Christopher Donnan, y Edward Lanning- o colaboraron con él, como Robert Benfer, Jeffrey Quilter y finalmente, y hasta el final de su carrera, Bernardino Ojeda. Además, y debido a la enorme extensión que cubrió con 
su trabajo de campo, sus interpretaciones de la antigüedad cuentan con elementos que en estos tiempos pocos pueden alcanzar.

Engel (1973), junto con otros investigadores pioneros como Lanning $(1963,1967)$ señalaron que las lomas, formadas por la niebla marina y que florecen de forma estacional a lo largo de la árida costa del Perú, fueron los primeros escenarios donde vivieron los cazadores-recolectores del Holoceno temprano y medio. Estos investigadores, además, sugirieron que las lomas fueron mucho más extensas que hoy. Engel argumentó que las lomas proveyeron abundantes recursos para los cazadores-recolectores trashumantes que vivieron allí durante los meses de invierno: lo que él llamó el "fog oasis situation" (1981:24). Engel y otros interpretaron las grandes extensiones de restos de plantas desecadas (como Tillandsia spp., ver Hesse 2012, 2014) y conchas de caracol fuera de los límites actuales de las lomas como evidencia de la mayor extensión de éstas en el pasado. Por su parte Lanning (1967:51), consideró que, desde la primera ocupación, en tiempos del Pleistoceno terminal, las lomas estuvieron en constante retroceso debido a los cambios de la corriente de Humboldt, lo que ha llevado a que solo se conserve la décima parte de la extensión de lo que en su momento existió, cuando fueron abandonadas abruptamente cerca de los 4500 AP.

Sin embargo, este modelo de Engel y Lanning sobre la ecología de las lomas para la prehistoria temprana del Perú fue rápidamente cuestionado como una "aventura transparente en determinismo ambiental” (Craig y Psuty 1968:126). Los críticos (Parsons 1970; Craig 1985, 1992) argumentaron, tal como Lynch (1971:140, nuestra traducción) resume, que:

"la vegetación y los animales de las lomas nunca pudieron haber sido importantes para el hombre, que la mayor extensión previa de vegetación de lomas nunca se ha establecido y que... no hay razón para creer que el clima de la costa del Perú haya sido sustancialmente diferente del presente durante los tiempos postglaciales".

Los extensos relictos de vegetación seca y caracoles fueron interpretados como producto de ocasionales perturbaciones climáticas del Fenómeno del Niño (ENOS) y no como prueba de que las lomas se extendieron de manera permanente fuera de su área actual. De hecho, algunas de estas formaciones extraordinariamente conservadas de Tillandsia desecada se remontan al Pleistoceno (Hesse 2014).

Algunos investigadores consideran que existe muy poca evidencia para sostener que hubo temporadas largas con cambios significativos del clima en la costa del Perú durante el Holoceno (p.ej. Craig y Psuty 1968; Parsons 1970; Craig 1985, 1992; Wells y Noller 1999). Estos invocaron otros factores para explicar los cambios en los patrones arqueológicos correspondientes al final del Precerámico, los cuales incluyen la excesiva explotación de ecozonas frágiles producto del crecimiento poblacional (Patterson y Moseley 1968:124; Weir y Derring 1986) y los cambios tecnológicos. Moseley (1975) argumentó que, en vez de sobreexplotar las lomas, las poblaciones concentraron sus esfuerzos en el uso intensivo de recursos marinos, actividad que fue posible gracias a la introducción de la agricultura del algodón. Asimismo, el modelo de Lanning fue señalado como insuficiente porque no consideró los efectos del aumento del nivel del mar en la conservación de los patrones arqueológicos observados en la costa (Richardson 1998), y nuevos datos sugerían que la adaptación marina es más longeva de lo que se presumía (Sandweiss 2009; Prieto y Sandweiss 2020). De todas formas, estos refinamientos recientes de las discusiones de la década de 1970 aún dejan sin respuesta la pregunta, ¿por qué las lomas de la costa peruana fueron abandonadas?

Entretanto, en los últimos años ha habido avances significativos en la investigación paleoclimática. Ahora se reconoce que hubo cambios climáticos a amplia escala en el Perú durante el largo retroceso de los glaciares que marcó la transición entre el Pleistoceno y el Holoceno, lo 
cual conllevó una progresiva estabilización del nivel del mar. Todo lo anterior pudo haber afectado significativamente la circulación de los monzones y las lluvias tropicales de origen amazónico en los Andes (p.ej. Mächtle y Eitel 2012).

Muchas reconstrucciones paleoclimáticas, utilizando datos (proxy) provenientes de diferentes puntos ubicados a través de los Andes, se han concentrado en la historia de las variaciones del Fenómeno del Niño y en sus efectos sobre la corriente de Humboldt a lo largo de la costa del Perú (Sandweiss et al. 1996; Rodbell et al. 1999; Moy et al. 2002; Sandweiss y Kelley 2012; Carré et al. 2014). En la misma costa peruana, donde los registros paleoclimáticos están ausentes o poco desarrollados (Sandweiss et al. 2020:1873), éstos a menudo involucran datos (proxy) recolectados de depósitos arqueológicos.

En este artículo, revisitaremos la controversia descrita acerca del rol de las lomas a la luz de los nuevos datos arqueológicos y ecológicos provenientes de las lomas de la costa sur, ${ }^{1}$ incluyendo la presentación de nuevos sitios arqueológicos documentados dentro de ellas. Nuestro trabajo sigue el camino trazado por Frédéric Engel para la arqueología de la costa sur, el cual comprende alguna de las más famosas manifestaciones culturales del Perú, como Paracas y Nazca; aunque sus predecesores precerámicos no continuaron siendo investigados (Isla 1990; Gorbahn 2020) desde los pioneros trabajos de Engel en la década de 1950.

Respecto al estudio de las lomas, a la fecha aún no se las comprende de forma adecuada y las pocas referencias a ellas (Parsons 1970; Oka y Ogawa 1984:115; Craig 1985) son inexactas y minimizan el potencial de sus recursos. La ausencia de investigaciones científicas puede explicarse porque las lomas de la costa sur se encuentran alejadas de poblados modernos y de la carretera Panamericana. Sin embargo, este "aislamiento" ha permitido que se preserve mejor su frágil biodiversidad, así como el registro arqueológico precerámico y, como veremos, coadyuva a fortalecer nuestras interpretaciones de la antigua ecología humana.

Usaremos nueva información para evaluar tanto la importancia de los recursos de las lomas en la ecología humana del Precerámico en una zona de la costa peruana que previamente no ha sido estudiada; como, en términos más amplios, la "capacidad de las lomas". Además, considerando las más recientes reconstrucciones paleoclimáticas del Holoceno de la costa sur (Carré et al. 2014), examinaremos la cuestión del "porqué" del cambio en el registro arqueológico a través del tiempo.

\section{LAS LOMAS DE LA COSTA SUR}

A lo largo de la costa peruana existen dos fuentes de agua que fluyen en diferentes momentos o estaciones del año. Una de ellas se origina a partir de una pequeña porción de las intensas lluvias de verano en el Amazonas que logra pasar el lado occidental de los Andes, recorriéndolo en forma de ríos y arroyos irregulares (Prohaska 1973). El segundo recurso es más efímero -al menos en los años que no ocurre el ENOS- y se presenta en invierno, entre los meses de agosto y noviembre, y se forma por las capas saturadas de humedad (neblina) originadas en los mares y empujadas al interior por los vientos. La neblina cubre la topografía del lado oeste de los Andes cuya altitud varía entre 300 a 1.000 m s.n.m. y en su camino desde el mar el fenómeno orográfico se va enfriando y generando lloviznas finas (garúas o camanchacas). La neblina es permanente debido a la reducida evaporación del estrato nuboso, el cual produce "precipitaciones pequeñas" y origina la formación de vegetación de niebla conocida como lomas. La vegetación actúa como una superficie que condensa y atrapa la niebla, y su crecimiento favorece el aumento de la cantidad de agua que se depositará sobre las laderas yermas (Engel 1973; Walter 1973; Oka 1986; Muenchow et al. 2013). Mientras esto ocurre, las formaciones de lomas florecen a lo largo del desierto con prados y pastos de un verde intenso. 
Coloquialmente el término "lomas" se utiliza en el Perú para referirse a la parte de la cordillera andina que se encuentra a lo largo de la costa del Pacífico. Debido a esto, ecólogos, geógrafos y arqueólogos han seguido utilizando el término de manera idiosincrática, tal como señala Craig (1985:28), para varios nichos ecológicos distintos, incluidos matorrales desérticos que se encuentran a mayor altitud y vegetación a lo largo de quebradas erráticas. En este documento usamos el término stricto sensu para referirnos a las comunidades de plantas que dependen exclusivamente de la neblina, compuestas gran parte del año por una reducida variedad de especies y dominada por la Tillandsia, pero que en los meses de invierno florece con un exuberante y efímero crecimiento herbáceo (Dillon et al. 2003).

Los oasis de neblinas pueden ser encontrados en buena parte de la costa oeste de América del Sur, entre los $6^{\circ} \mathrm{S}$ y $30^{\circ} \mathrm{S}$ (Manrique et al. 2010). En el Perú al menos 70 localidades cuentan con lomas y en total cubren un área de $8.000 \mathrm{~km}^{2}$ (Dillon et al. 2003; Dillon 2011). Su comunidad botánica delimita claramente un ecosistema único en la ecología y composición florística de América del Sur (Stafford 1939; Dillon 2011) y está compuesta de una variedad de especies de ciclo anual de corta vida y de arbustos, los cuales suman un total de 850 especies, divididos en 385 géneros y 83 familias (Dillon 2011). Además, las lomas comprenden una variedad extensa de vida animal (Zeballos et al. 2000).

Aproximadamente el $40 \%$ de las especies vegetales de las lomas de la costa sur pueden ser clasificadas como plantas perennes y se mantienen durante la temporada seca gracias al almidón vegetal almacenado en órganos de carácter subterráneo ("geofito", figura 5B y C) como las raíces, tubérculos y bulbos (en este último caso, el 15\% de ellos son monocotiledóneas). De hecho, nosotros encontramos que muchas de las plantas clasificadas como de ciclo anual, fueron en realidad perennes, creciendo de raíces profundas o, simplemente, volviendo a brotar de tallos con hojas aparentemente secas, pero que mantienen sus raíces.

Cuando retornan las nieblas de invierno, y dependiendo de la densidad y duración de la estación, las plantas perennes vuelven a brotar, y con ello aumenta la captura de la humedad del ambiente, ayudando a germinar a las semillas de plantas anuales depositadas el año anterior. Con la llegada de la primavera y el aumento de la radiación solar, toda la loma florece de manera simultánea para maximizar la polinización rápida y formar semillas, muchas de las cuales están adaptadas para resistir las altas temperaturas de verano y están esparcidas en toda la superficie del desierto esperando el regreso de la niebla de invierno.

La vegetación de las lomas es altamente sensible a los cambios climáticos del Fenómeno del Niño y, de hecho, las plantas de las lomas dependen de la periódica recurrencia de El Niño para propagarse (Cano et al. 1999; Manrique et al. 2010; Dillon 2011; Kalicki et al. 2014). Sin embargo, es importante anotar que nuestra investigación observó que la relación entre la productividad de las lomas y el fenómeno climático del Oscilación del Sur-El Niño (ENOS) es bastante compleja, sobre todo porque el mismo ENOS se presenta en diversas formas y con diferentes impactos locales: mientras que algunas partes de las lomas fueron favorecidas por las variaciones climáticas producidas con el ENOS, otras mostraron un deterioro. Más adelante ampliaremos con respecto a estas implicancias.

Dentro de las lomas se pueden distinguir grupos cuya composición está determinada por la cantidad de humedad disponible, que depende de la altitud, pero también de la relación de la topografía y textura de la superficie del terreno con la intensidad, dirección y velocidad de la niebla costera (Ono 1986; Muenchow et al. 2013). Las más diversas praderas herbáceas florecen con las nieblas invernales en un cinturón inmediatamente por debajo de la altitud en la que la capa de inversión intercepta la tierra (Muenchow et al. 2013). A lo largo de estas márgenes, específicamente en los lugares donde las condiciones son más áridas, las lomas de la costa sur se caracterizan por ser la transición entre los espacios con baja densidad de cactus, y las zonas 
de alta densidad de especies de Tillandsia, los cuales pueden extenderse por vastas porciones del desierto (Whaley et al. 2010; Hesse 2012).

A diferencia de las lomas presentes a lo largo de la costa peruana, las de la costa sur están separadas del núcleo de la cordillera de los Andes por una meseta formada por capas sedimentarias que cubren cerca de $60 \mathrm{~km}$ de ancho (figura 1). Estas porciones del batolito de granito se extienden a lo largo de los casi $150 \mathrm{~km}$ de la costa peruana y se levantan del océano abruptamente para alcanzar alturas de alrededor de 800 m, 1000 m y 1791 m s.n.m. en las lomas de Morro Quemado, Ullujaya y Amara, y San Fernando, respectivamente. Además, las lomas de la costa sur se encuentran aledañas al litoral y bastante lejos de los cursos del río Ica, que fluye de norte a sur, y, más al oeste, del río Grande de Nazca con sus varios afluentes.

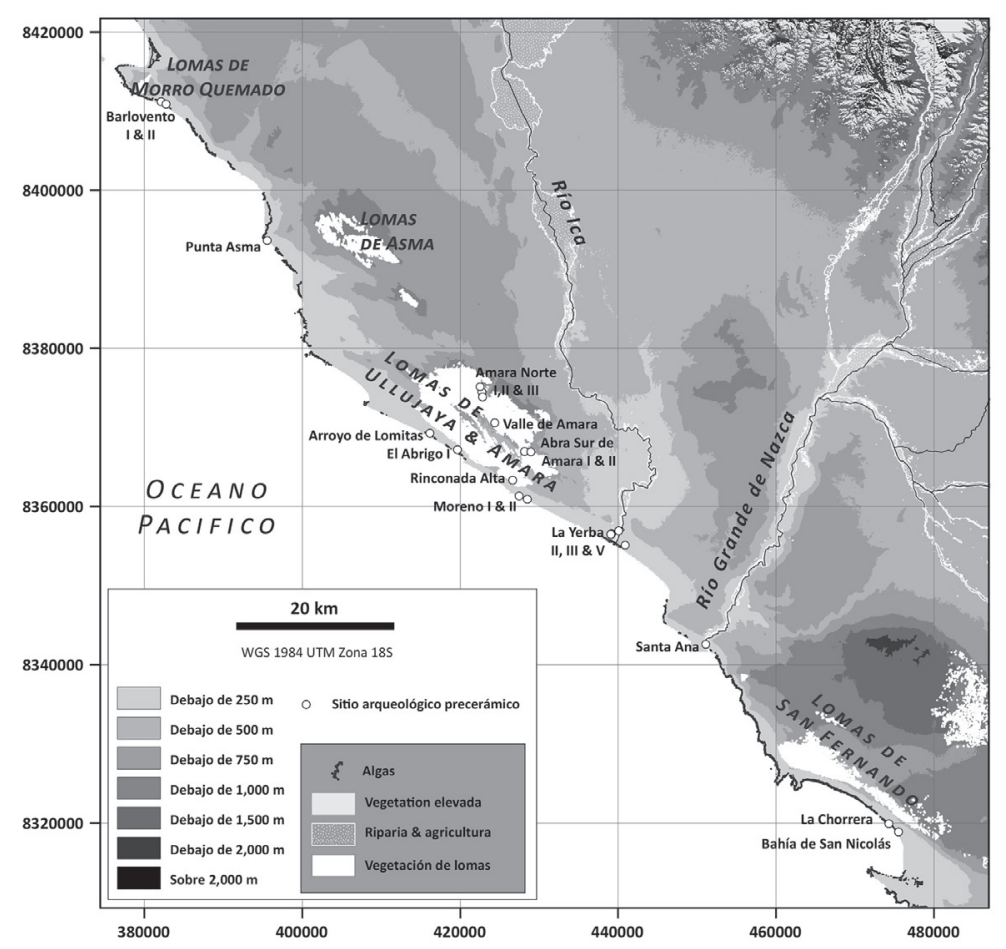

Figura 1. Mapa de la costa sur que muestra los sitios precerámicos junto con lomas y otras asociaciones de vegetación

\section{NUEVAS INVESTIGACIONES ARQUEOLÓGICAS Y ECOLÓGICAS}

El One River Project de la Universidad de Cambridge y el Royal Botanical Gardens Kew (RGB Kew) colaboraron, desde el 2012 hasta el 2014, de manera estrecha y ejecutando diferentes tareas de trabajo de campo:

1. RGB Kew realizó un análisis de la distribución espacial de la vegetación y la composición florística de las lomas de San Fernando y Amara, recolectando especies herbarias sistemáticamente y elaborando mapas de vegetación utilizando como fuentes imágenes Landsat, correspondientes al 8 de marzo de 2014 a marzo de 2015, las cuales fueron complementadas con imágenes de GeoEye, de octubre/noviembre de 2011, para la región de San Fernando.

2. El One River Project de la Universidad de Cambridge realizó investigaciones de la arqueolo- 
gía precerámica asociada con las lomas de la costa sur (Beresford-Jones et al. 2015a). Engel (1981, 1991) identificó 14 sitios precerámicos a lo largo de la línea del litoral que se extiende entre Morro Quemado y la Bahía de San Nicolás, de los cuales ocho fueron fechados con radiocarbono. Además de investigar con más detalle los sitios registrados por Engel, así como recolectar muestras, dirigimos nuestros esfuerzos a los sitios que se encuentran al interior de las lomas. En la mayoría de los sitios se registraron los perfiles expuestos, se realizaron nuevas excavaciones y se recolectó material de superficie. Durante los trabajos de campo se tomaron muestras para flotación -de donde se extrajeron restos orgánicos y macrorrestos-, análisis geoquímicos, de radiocarbono, etc.

Las figuras 2 y 8 , presentan los resultados del reconocimiento arqueológico hecho en la zona hasta la fecha, mostrando el paisaje precerámico que compone la sección de la costa sur que se ubica entre Morro Quemado y la Bahía San Nicolás. Los fechados que acompañan (tabla 1) fueron calibrados usando la curva de ShCal20 (Hogg et al. 2020) en OxCal versión 4.4.4 (Bronk Ramsey 2009).

Tabla 1

\begin{tabular}{|c|c|c|c|c|c|c|c|c|c|}
\hline \multirow{3}{*}{$\begin{array}{l}\text { Sitio } \\
\text { Amara } \\
\text { Norte I }\end{array}$} & \multirow{3}{*}{$\begin{array}{c}\begin{array}{c}\text { Código del } \\
\text { sitio }\end{array} \\
\text { ORP-585 }\end{array}$} & \multirow{3}{*}{\begin{tabular}{|c|}
$\begin{array}{c}\text { Lomas } \\
\text { asociada }\end{array}$ \\
$\begin{array}{c}\text { Amara- } \\
\text { Ullujalla }\end{array}$ \\
\end{tabular}} & \multirow{3}{*}{$\begin{array}{c}\text { Material } \\
\text { fechado }\end{array}$} & \multicolumn{5}{|c|}{ Fechas de Radiocarbono } & \multirow{3}{*}{$\begin{array}{c}\text { Referencia } \\
\text { Beresford- } \\
\text { Jones et al. } \\
2015\end{array}$} \\
\hline & & & & \multirow{2}{*}{\begin{tabular}{|l|}
$\begin{array}{c}{ }^{14} \mathbf{C} \\
\text { años }\end{array}$ \\
\cline { 1 - 1 } \\
4325
\end{tabular}} & \multirow{2}{*}{\begin{tabular}{|c|}
$\begin{array}{c}\text { Error } \\
(+/-)\end{array}$ \\
30
\end{tabular}} & \multicolumn{2}{|c|}{ Cal AP $\mathbf{P}^{(1)}$} & \multirow{2}{*}{$\begin{array}{c}\begin{array}{c}\text { Código de } \\
\text { laboratorio }\end{array} \\
\begin{array}{c}\text { OxA- } \\
32350\end{array} \\
\end{array}$} & \\
\hline & & & & & & 4966 & 4730 & & \\
\hline $\begin{array}{l}\text { Arroyo } \\
\text { de } \\
\text { Lomitas }\end{array}$ & $15 \mathrm{a}$ VI-525 & $\begin{array}{l}\text { Amara- } \\
\text { Ullujalla }\end{array}$ & $\begin{array}{c}\text { No } \\
\text { especificado }\end{array}$ & 4360 & 95 & 5290 & 4618 & $\mathrm{I}-7774$ & $\begin{array}{c}\text { Engel } \\
1991: 56\end{array}$ \\
\hline \begin{tabular}{|l|} 
El \\
Abrigo
\end{tabular} & $15 \mathrm{a}$ VI-550 & $\begin{array}{c}\text { Amara- } \\
\text { Ullujalla }\end{array}$ & $\begin{array}{c}\text { No } \\
\text { especificado }\end{array}$ & 8835 & 145 & 10204 & 9540 & I-7772 & $\begin{array}{c}\text { Engel } \\
1991: 56\end{array}$ \\
\hline $\begin{array}{l}\text { La } \\
\text { Yerba I }\end{array}$ & $15 \mathrm{~b}$ VII-50 & $\begin{array}{c}\text { Amara- } \\
\text { Ullujalla }\end{array}$ & 'Plantas' & 7520 & 300 & 9018 & 7693 & Birm-511 & $\begin{array}{c}\text { Engel } \\
\text { 1991: } 56\end{array}$ \\
\hline $\begin{array}{l}\text { La } \\
\text { Yerba } \\
\text { I (UE } \\
1024) \\
\end{array}$ & ORP-209 & $\begin{array}{l}\text { Amara- } \\
\text { Ullujalla }\end{array}$ & Carbón & 6375 & 39 & 7419 & 7162 & $\begin{array}{l}\text { OxA- } \\
30913\end{array}$ & $\begin{array}{l}\text { Beresford- } \\
\text { Jones et al. } \\
\quad 2015\end{array}$ \\
\hline \begin{tabular}{l|} 
La \\
Yerba II
\end{tabular} & $\begin{array}{c}15 b \text { VII-45 } \\
\& 100\end{array}$ & $\begin{array}{l}\text { Amara- } \\
\text { Ullujalla }\end{array}$ & Carbón & 6470 & 110 & 7572 & 7075 & I-7773 & $\begin{array}{c}\text { Engel } \\
1991: 56\end{array}$ \\
\hline \begin{tabular}{|l|} 
La \\
Yerba \\
II (UE \\
$1015)$ \\
\end{tabular} & ORP-207 & $\begin{array}{l}\text { Amara- } \\
\text { Ullujalla }\end{array}$ & $\begin{array}{l}\text { Rizoma de } \\
\text { Scirpus sp. }\end{array}$ & 5988 & 33 & 6887 & 6672 & $\begin{array}{l}\text { OxA- } \\
29975\end{array}$ & $\begin{array}{l}\text { Beresford- } \\
\text { Jones et al. } \\
\quad 2015\end{array}$ \\
\hline $\begin{array}{l}\text { La } \\
\text { Yerba } \\
\text { II (UE } \\
1079) \\
\end{array}$ & ORP-208 & $\begin{array}{l}\text { Amara- } \\
\text { Ullujalla }\end{array}$ & $\begin{array}{l}\text { Cáscara de } \\
\text { maté } \\
\text { (Lagenaria } \\
\text { sp.) }\end{array}$ & 6029 & 32 & 6947 & 6735 & $\begin{array}{l}\text { OxA- } \\
29944\end{array}$ & $\begin{array}{c}\text { Beresford- } \\
\text { Jones et al. } \\
\quad 2015\end{array}$ \\
\hline \begin{tabular}{l|} 
La \\
Yerba \\
II (nivel \\
5)
\end{tabular} & ICA-IN & $\begin{array}{l}\text { Amara- } \\
\text { Ullujalla }\end{array}$ & Carbón & 5940 & 45 & 6884 & 6567 & OS-60556 & $\begin{array}{l}\text { Carré et } \\
\text { al. 2014: } \\
\text { Tabla S2 }\end{array}$ \\
\hline \begin{tabular}{l|} 
La \\
Yerba \\
III
\end{tabular} & $15 \mathrm{~b}$ VII-55 & $\begin{array}{l}\text { Amara- } \\
\text { Ullujalla }\end{array}$ & 'Plantas' & 5430 & 140 & 6493 & 5895 & Birm-513 & $\begin{array}{c}\text { Engel } \\
1991: 56\end{array}$ \\
\hline
\end{tabular}


Relaciones de la Sociedad Argentina de Antropología 46 (2), julio-diciembre 2021: 383-413

(Tabla 1. Continuación)

\begin{tabular}{|c|c|c|c|c|c|c|c|c|c|}
\hline \multirow[b]{2}{*}{ Sitio } & \multirow{2}{*}{$\begin{array}{l}\text { Código del } \\
\text { sitio }\end{array}$} & \multirow{2}{*}{$\begin{array}{c}\text { Lomas } \\
\text { asociada }\end{array}$} & \multirow{2}{*}{$\begin{array}{l}\text { Material } \\
\text { fechado }\end{array}$} & \multicolumn{5}{|c|}{ Fechas de Radiocarbono } & \multirow[b]{2}{*}{ Referencia } \\
\hline & & & & $\begin{array}{c}{ }^{14} \mathrm{C} \\
\text { años }\end{array}$ & $\begin{array}{c}\text { Error } \\
(+/-)\end{array}$ & Cal & $\mathbf{P}^{(1)}$ & $\begin{array}{c}\text { Código de } \\
\text { laboratorio }\end{array}$ & \\
\hline $\begin{array}{l}\text { La } \\
\text { Yerba } \\
\text { III (UE } \\
9020)\end{array}$ & $15 \mathrm{~b}$ VII-55 & $\begin{array}{l}\text { Amara- } \\
\text { Ullujalla }\end{array}$ & $\begin{array}{c}\text { Frijol de } \\
\text { pallar } \\
\text { (Phaseolus } \\
\text { lunatus) }\end{array}$ & 5381 & 33 & 6275 & 5999 & $\begin{array}{l}\text { OxA- } \\
32290\end{array}$ & $\begin{array}{c}\text { Beresford- } \\
\text { Jones et } \\
\text { al. } 2015 \text {, } \\
2018\end{array}$ \\
\hline $\begin{array}{l}\text { La } \\
\text { Yerba } \\
\text { III (UE } \\
9009) \\
\end{array}$ & $15 b$ VII-55 & $\begin{array}{l}\text { Amara- } \\
\text { Ullujalla }\end{array}$ & $\begin{array}{c}\text { Poste de } \\
\text { madera } \\
\text { (Salix sp.) }\end{array}$ & 5290 & 33 & 6181 & 5925 & $\begin{array}{l}\text { OxA- } \\
32291\end{array}$ & $\begin{array}{c}\text { Beresford- } \\
\text { Jones et al. } \\
\quad 2015\end{array}$ \\
\hline $\begin{array}{l}\mathrm{La} \\
\text { Yerba } \\
\mathrm{V}^{(2)}\end{array}$ & $15 \mathrm{~b}$ VII-25 & $\begin{array}{l}\text { Amara- } \\
\text { Ullujalla }\end{array}$ & Carbón & 6150 & 120 & 7266 & 6676 & $\mathrm{I}-3560$ & $\begin{array}{c}\text { Engel } \\
1991: 56\end{array}$ \\
\hline $\begin{array}{l}\text { Santa } \\
\text { Ana }\end{array}$ & $\begin{array}{c}15 b \text { VII-8 } \\
\& 19\end{array}$ & Mainsa & $\begin{array}{c}\text { 'Fardo } \\
\text { funerario' }\end{array}$ & 4720 & 120 & 5656 & 4980 & - & $\begin{array}{c}\text { Engel } \\
1981: 61\end{array}$ \\
\hline $\begin{array}{l}\text { Bahía } \\
\text { de San } \\
\text { Nicolás }\end{array}$ & $16 \mathrm{aII}-40$ & $\begin{array}{c}\text { San } \\
\text { Fernando }\end{array}$ & $\begin{array}{c}\text { No } \\
\text { especificado }\end{array}$ & 5560 & 80 & 6497 & 6020 & - & $\begin{array}{c}\text { Engel } \\
1981: 59\end{array}$ \\
\hline
\end{tabular}

\section{ECOLOGÍA HUMANA EN LA ÉPOCA PRECERÁMICA, COSTA SUR DEL PERÚ}

Los patrones del registro arqueológico precerámico de la costa sur reflejan la distribución de los diferentes recursos alimenticios, combustible y de materia prima. Con todo, el recurso primario que determina dónde se asentó la gente y por dónde transitaron dentro de un paisaje extremadamente árido fue, por supuesto, el agua (Erlandson 2001). Esto último se observa claramente en la figura 1, en donde se puede ver que los sitios arqueológicos precerámicos más grandes y visibles se encuentran próximos a la boca del río Ica y Nazca, mientras que los otros sitios que se encuentran lejos del curso de los ríos están íntimamente asociados con las lomas. De hecho, como veremos, todos estos sitios precerámicos, independientemente de su ubicación, contienen importantes recursos derivados de las lomas.

\section{Sitios ubicados cerca al estuario}

Los sitios arqueológicos más grandes y visibles que se ubican entre Morro Quemado y la bahía de San Nicolás se encuentran cerca de las bocas de los ríos Ica y Nazca. Algunos de los ubicados próximo al estuario del río Ica datan de la etapa temprana del periodo Precerámico Medio (tabla 1 y figura 8), entre ellos, los más grandes y visibles son La Yerba II y La Yerba III. La estratificación de los mencionados sitios está compuesta de una alta densidad de restos asociados con la caza, la pesca y la recolección de recursos marinos: mamíferos marinos, aves, peces y moluscos, particularmente las machas (Mesodesma donacium), que hasta El Niño de 1997-98 abundaban cerca de la línea del litoral de las playas. En las capas también se observan restos de vegetales muy bien preservados, inter alia, tenemos: algas, rizomas de Cyperacea; petates, postes con restos de esteras como vestigios de casas, líneas y redes elaboradas con fibras de plantas no domesticadas y botellas de calabaza silvestre (Lagenaria sp., fechado en 6947-6735 años cal AP, OXA-29944, tabla 1), y pallares domesticados (Phaseolus lunatus, fechado en 6275-5999 años cal AP, OXA-32290, tabla 1). Estos últimos posiblemente cultivados en la ribera del río. Los sitios 

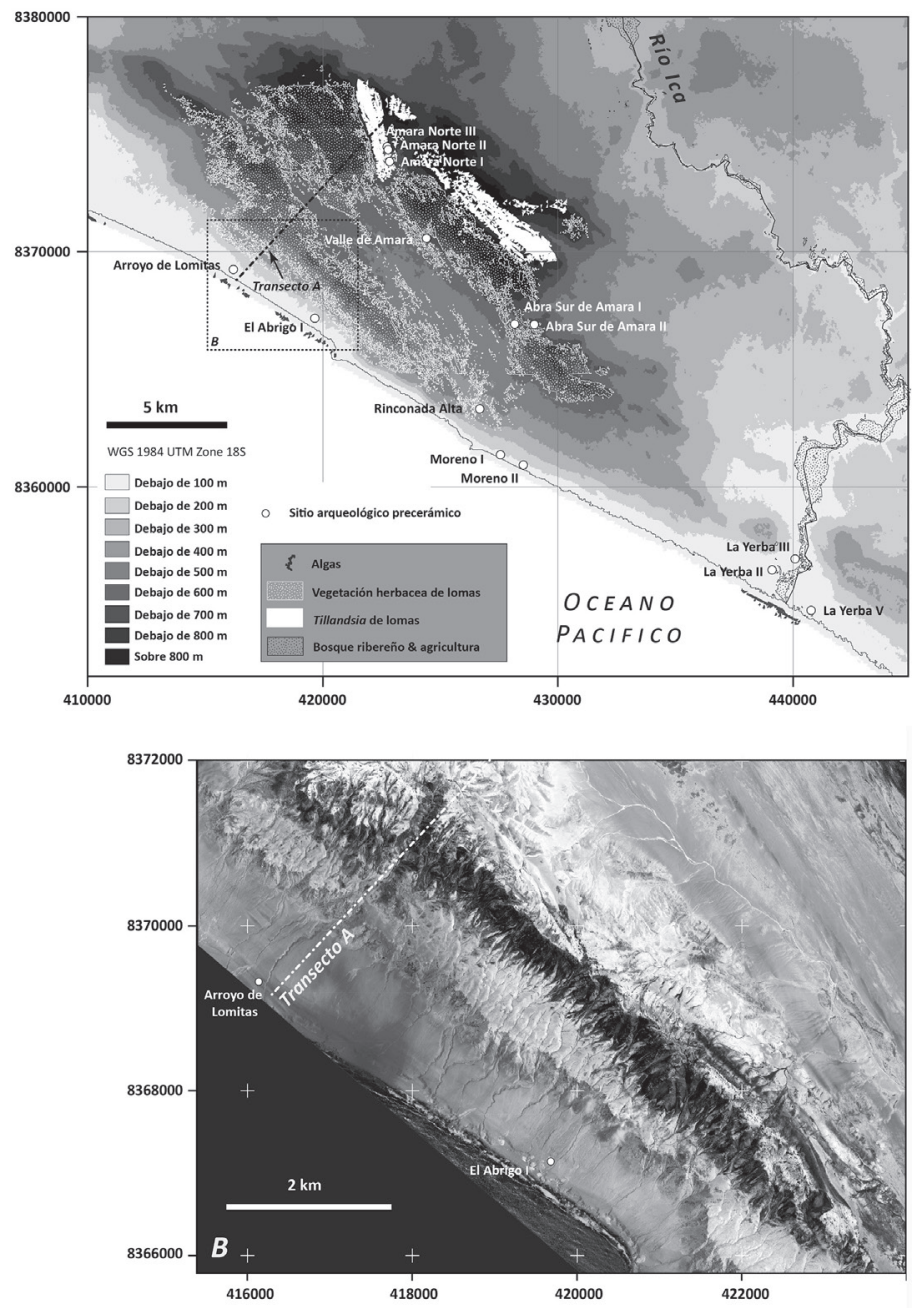

Figura 2. Mapa de las lomas de Amara y Ullujaya que muestra los sitios precerámicos junto con las asociaciones de vegetación de lomas y el Transecto A a través del cual RBG Kew realizó recolecciones sistemáticas de plantas en parcelas. El recuadro B muestra un detalle de las lomas de Amara usando NIR para mostrar la alta densidad de la vegetación de las lomas como gris oscuro el 10 de febrero de 2010 durante un episodio de El Niño moderado

ubicados cerca del estuario han sido interpretados como campamentos base (sensu Binford 1980) de cazadores-recolectores de movimiento estacional. Sin embargo, es importante anotar que en La Yerba III existe evidencia propia del estilo de vida sedentario (Beresford-Jones et al. 2018, 2021). La ubicación estratégica en el punto de "confluencia" de diferentes hábitats: estuarios, playas arenosas y rocosas, y lomas ecológicamente diversas; habría proporcionado, de manera estacional y predecible, abundantes recursos que pudieron ser aprovechados por los diferentes miembros de los grupos humanos (hombre, mujeres y niños) en distintos momentos del año (Moseley 1975).

Tierra adentro de los estuarios, se reportan pocos sitios del Precerámicos Medio en la costa sur (Isla 1990; Vogt 2011; Gorbahn 2020), aunque sin duda otros restos han sido tapados por posteriores cambios agrícolas y geomorfológicos. Sin embargo, mucho más allá de los cursos de los ríos, nuestras investigaciones revelan otros componentes del paisaje del Precerámico Medio: 
todos los cuales dependen directamente de las lomas para proporcionar recursos clave, como el agua y combustible. Estos sitios son de dos tipos generales.

\section{Los sitios ubicados en el litoral adyacente a las lomas}

Este primer grupo está compuesto por los sitios Arroyo de Lomitas, Abrigo I y Bahía de San Nicolás, todos identificados originalmente por Engel (1981), y localizados en el límite entre las lomas y el mar, de modo tal que sus ocupantes tuvieron acceso simultáneo tanto a los recursos marinos como a aquellos propios de las lomas (figura 1 y 4 ).

Durante la documentación de la estratificación del sitio Arroyo de Lomitas registramos densas capas y fogones delimitados con piedras (figura $3 \mathrm{E}$ y F), lo que nos sugiere que hubo una fuente de agua que pudo ser aprovechada por un pequeño grupo de cazadores-recolectores durante temporadas cortas. La estratificación del Arroyo de Lomitas, y los otros sitios, cuentan con abundantes recursos marinos propios de las playas rocosas adyacentes, particularmente los gasterópodos, mejillas y algas. Es llamativo que hasta hoy los pescadores itinerantes conozcan el sitio como "Agua Dulce" (o "Pozo de los Chilenos", como se puede leer en el plano del Instituto Geográfico Nacional Peruano, Sheet 1741) y afirmen que es posible obtener agua excavando un pozo. Es conocido, que todos los sitios que se encuentran a lo largo de la costa están asociados a fuentes de agua que nacen de las lomas (Strong 1957:8; Engel 1981, 1991:85).
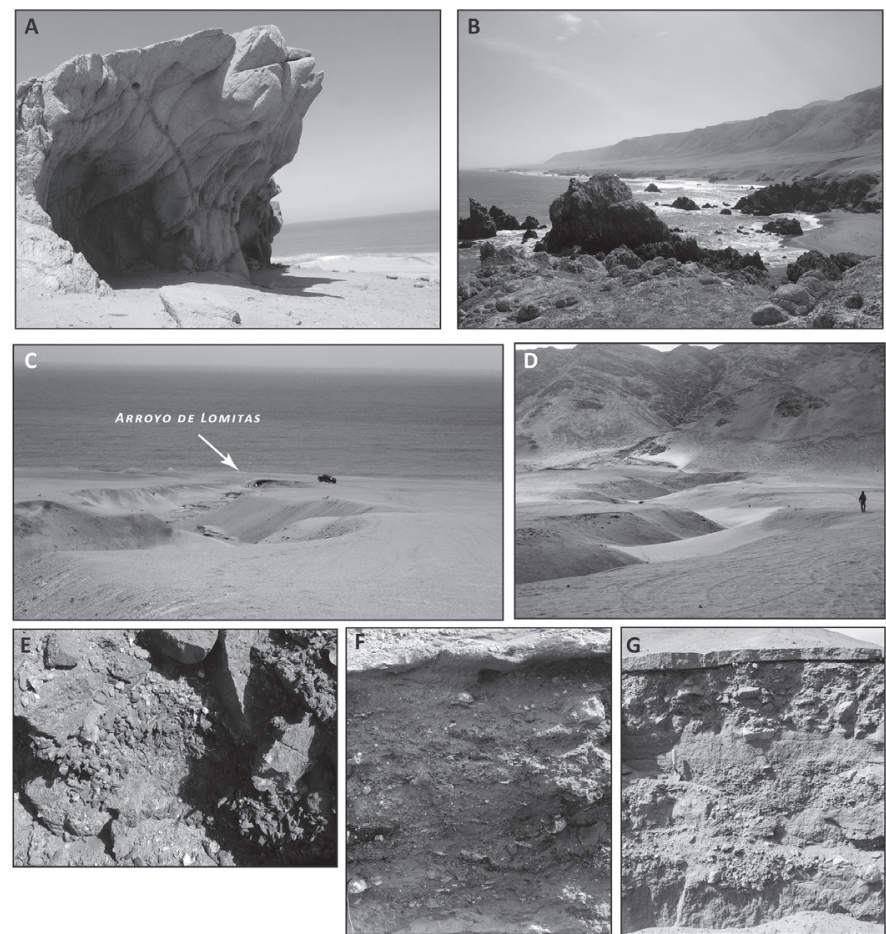

Figura 3. Detalles de los sitios precerámicos a lo largo de la costa de Amara: A) sitio Precerámico Temprano El Abrigo (10204-9540 Cal AP); B) vista norte de la playa que limita con Lomas de Amara; C) y D) vista oeste hacia el mar y este hacia las lomas de Amara, respectivamente, del curso de agua que pasa por el sitio Precerámico Medio Arroyo de Lomitas (5290-4618 Cal AP); E) detalle del carbón

en una fogata de Arroyo de Lomitas; F) sección de Arroyo de Lomitas mostrando capas de basura y concentraciones de carbón; G) perfil de Arroyo de Lomitas mostrando depósitos clasiticos y aluvionales 
La cordillera en la costa es una masa de granito de topografía abrupta e impermeable que alcanza los 1000 m s.n.m. y forma una superficie plana y favorable para los procesos de condensación y formación de niebla. En las superficies de las Lomas de Amara y San Fernando se aprecian huellas de múltiples arroyos, a manera de profundas incisiones sobre la terraza marina del Holoceno, lo cual es evidencia del recorrido periódico de agua. La presencia de material clástico en los depósitos acumulados por los arroyos sería, quizá, evidencia de la temporalidad y del gran dinamismo de los Fenómenos del Niño ocurridos (figura 3G). Incluso, algunas capas delgadas atestiguan el fluir de agua en años normales y sus cursos cuentan con especies de vegetales perennes como Atriplex rotundifolia (Chenopodiaceae), Croton alnifolius (Euphorphiaceae) y Tiquilia spp. (Boraginaceae), y una variedad de pequeñas hierbas tales como Alternanthera sp. (Amaranthaceae) y Parietaria denilis (Urticaceae), y grandes relictos de cactus Carryaocactus aff. Brochypetalus, cuyos tamaños son evidencia significativa de la disponibilidad del agua.

A diferencia de las lomas costeras, donde están presentes los dos tipos de fuentes de agua descritas arriba, aquí, solo es posible obtener agua de la condensación de las nieblas marinas. Esos cursos de arroyo, alimentados por las nieblas, habrían permitido la ocupación humana precerámica a lo largo del litoral árido y lejos del curso de los ríos (y estuarios), pero en la proximidad inmediata tanto de lomas como de recursos marinos particulares.

\section{Campamentos logísticos en el interior de las lomas}

Engel tuvo mucho éxito en registrar y ubicar sitios precerámicos a lo largo de la costa, aunque este mismo éxito no lo acompañó para encontrar asentamientos humanos dentro de las lomas de la costa sur (Engel 1981:27; la sorpresa es mayor si consideramos que la Lomas de Amara solas cubren $400 \mathrm{~km}^{2}$ ). Sin embargo, tales sitios existen, conspicuos en gran parte como grandes acumulaciones de conchas de caracoles terrestres (figuras 1 y 5).

En las lomas, la preservación de los restos orgánicos es muy reducida debido a la acción de la humedad del invierno sobre el material. En otros lugares de lomas, Craig (1992) interpreta acumulaciones similares de caracoles terrestres como conjuntos de muerte natural. Sin embargo, para nuestro caso, el origen humano de Amara Norte I es indiscutible por la estratificación de los contextos (figura 4E), que cuentan con restos de conchas marinas y erizos de mar, piedras de moler (figura 4F) y un fogón fechado en 4966-4730 años cal AP (OxA-32350, tabla 1). Alrededor del "conchal" se encuentran restos de puntas de proyectil de obsidiana (figura 4B) y desechos de reducción de preformas expuestas en superficie por acción del viento.

Otros sitios semejantes identificados durante nuestra investigación en las lomas de Amara son Amara Norte, Valle de Amara y Abra Sur. Ellos habrían cumplido la función de campamento logístico: ocupados por corto tiempo y de forma estacional para cazar y recolectar recursos especifico de las lomas, particularmente plantas geofitas y caracoles de tierra. En la arqueología, estos tipos de sitios son raros, pero no desconocidos (p.ej. Engel 1981; Larrain et al. 2001).

En ocasiones, inesperadas precipitaciones forman estanques de corta vida en las lomas de San Fernando y Amara. En tiempos históricos, el agua fue "cosechada" de las neblinas utilizando tejidos, los cuales permiten recolectar cerca de 10 litros por $\mathrm{m}^{2}$ cada día (Klemm et al. 2012). No se cuenta con evidencia para demostrar si en el Precerámico Medio (8000-5000 años cal AP) se conocía la tecnología de recolección de agua con tejido, pero durante la estación de invierno -momento de mayor productividad de las lomas- en ocasiones el goteo de la neblina que es recogida en las rocas puede alcanzar cantidades de agua significativa (Larrain et al. 2001).

No existe duda de que durante el Precerámico Medio el agua dulce fue transportada utilizando botellas de calabaza o la piel de animales: restos de botellas fueron recuperados de los campamentos bases ubicados en la desembocadura. En consideración de nuestras observaciones 


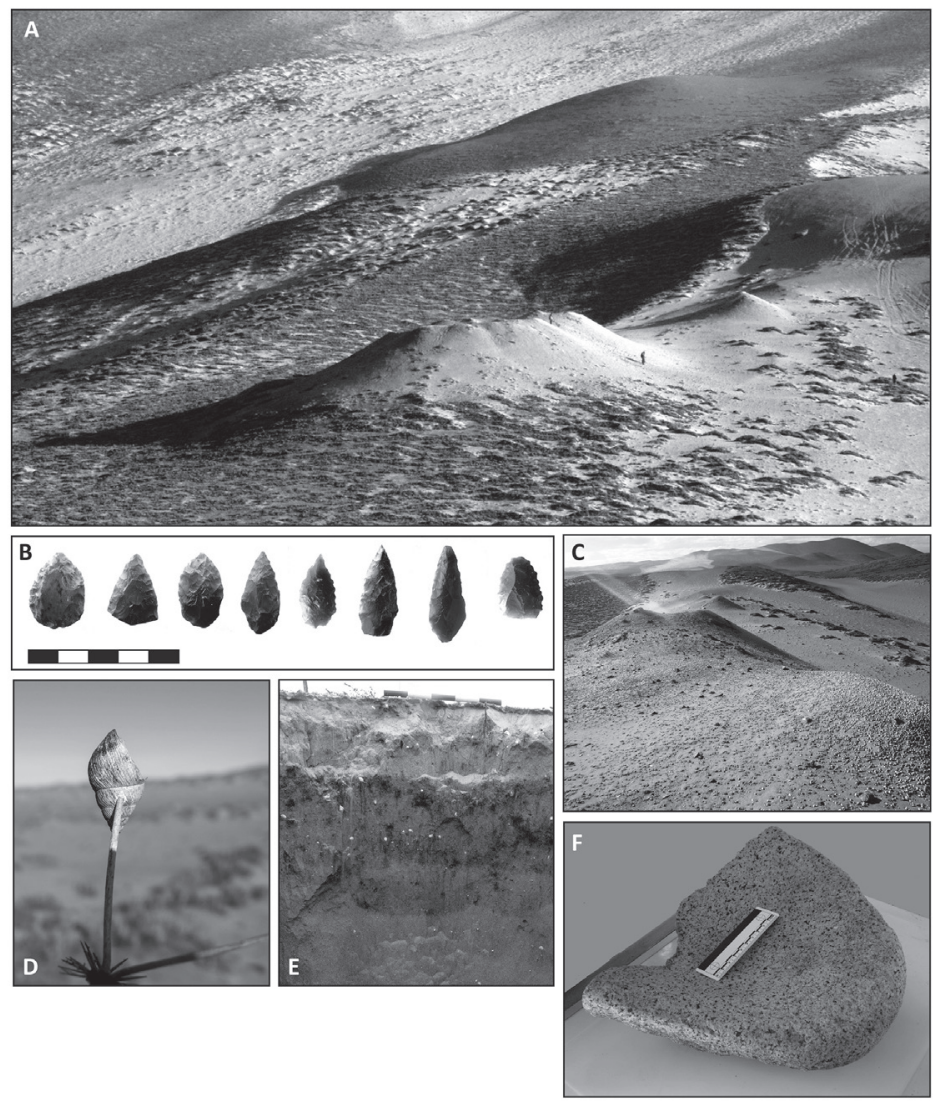

Figura 4. Detalles del sitio Precerámico Amara Norte I en el corazón de lomas de Amara: A) vista norte del sitio; B) selección de puntas de proyectil de obsidiana, Amara Norte I; C) vista sur de Amara Norte I con concentración superficial de conchas de Bostryx; D) concha de Bostryx conspersus que muestra orificio de punción correspondiente a la presunta extracción de carne con espina de cactus; E) sección de excavación de Amara Norte I mostrando conchas y carbón aglomerados fechado en 4966-4730 cal AP;

F) mortero de piedra ('batan') de Amara Norte I

de la hidrología de las lomas y la ubicación de los sitios arqueológicos del Precerámico (figuras 1 y 2), algunos en lugares muy alejados del curso del río (figuras $3 \mathrm{~F}$ y $5 \mathrm{~A}$ ), sugerimos que el agua "originada" de la neblina de la costa fue suficiente para sostener a pequeños grupos de cazadores-recolectores por corto tiempo. En el siguiente apartado evaluaremos el potencial de los recursos que brindan las lomas a partir de nuestras investigaciones (ecológicas y arqueológicas) en las lomas de la costa sur.

\section{La fauna de las lomas}

A pesar del carácter efímero y estacional de la vegetación de las lomas de la costa sur, este es hogar de una variedad de especies de animales, la cual incluye grandes mamíferos como el guanaco (Lama guanicoe, Palomino 2006), dos especies de zorros (Lycalopex spp.), roedores, lagartijas y aves, además de invertebrados como insectos y caracoles. Los restos de estos últimos son los elementos más importantes y frecuentes propios de las lomas que componen la estrati- 
ficación de los sitios del periodo Precerámico Medio de la costa sur, situación que evidencia su importancia en la dieta humana (Reitz 2003; Beresford-Jones et al. 2015b; Gorbahn 2020).
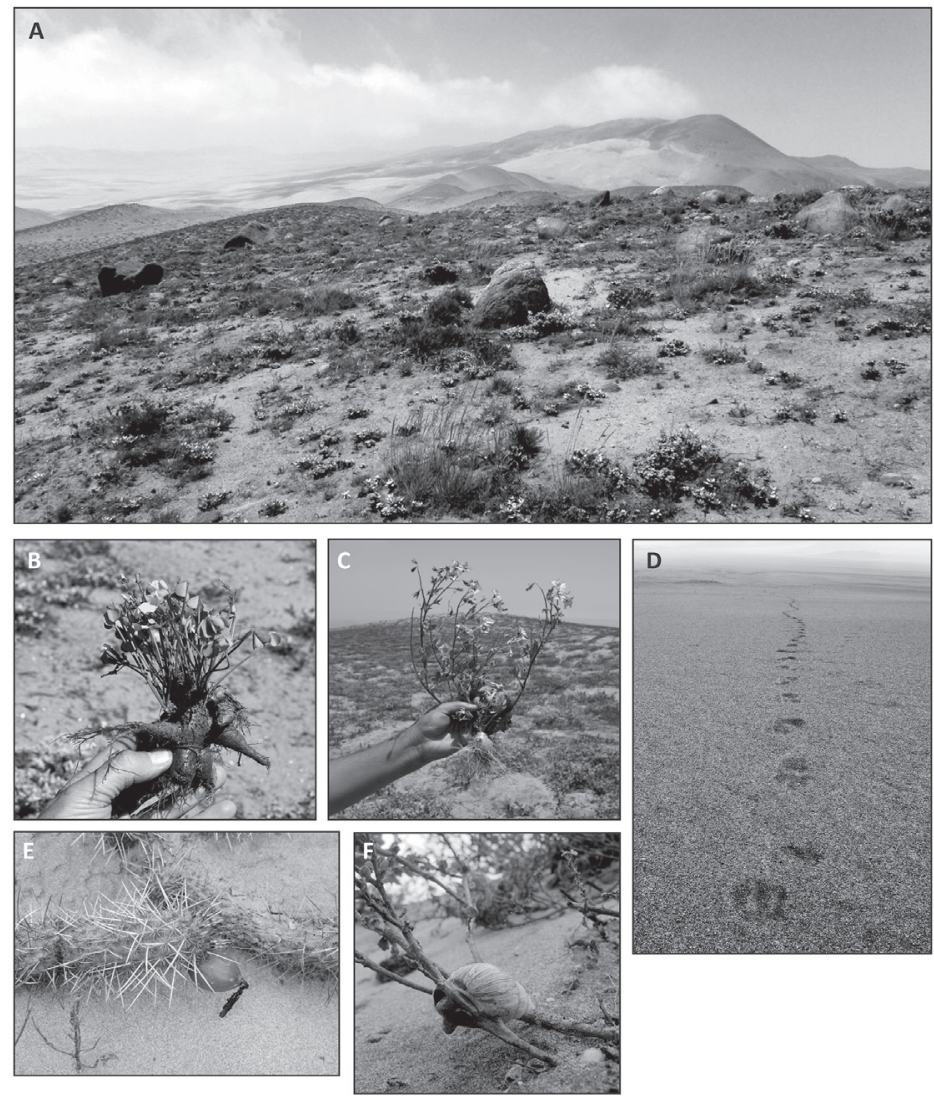

Figura 5. Recursos de las lomas de la costa sur: A) lomas de Amara, agosto 2013; B) y C) tubérculos de lomas de Amara, Oxalis sp. y Solanum montanum, respectivamente; D) huellas de guanaco (Lama guanicoe), lomas de Amara; E) fruta comestible de cactus Haageocereus sp., lomas de San Fernando;

F) caracol Bostryx conspersus vivo en Atriplex sp., lomas de Amara

Actualmente, en las lomas de Ica se encuentran dos especies de caracoles de los cuales el más común es el que nosotros identificamos tentativamente como Bostyrx reentsi (Philippi 1851; Bram Breure, comunicación personal). Los caracoles entran en fase activa cuando las lomas están en su etapa más productiva (figura 6G), e "invernan" en grupos durante los meses secos (Ramírez 1988; Ramírez et al. 2003). Hemos observado caracoles vivos en la vegetación de las lomas, pero también en los límites de la ecozona, donde se alimentan de las algas que crecen sobre las Tillandsia e "invernan" en sus tallos o debajo de sus raíces.

Los sitios arqueológicos como Amara Norte y Abra Sur de Amara, los cuales se ubican próximos a concentraciones de Tillandsia en las lomas de Amara entre 550 y 700 m s.n.m., tienen capas arqueológicas que presentan grandes cantidades de conchas de caracol (figura 5C y D). Mientras tanto, sitios a lo largo del litoral como Arroyo de Lomitas, Abra I y La Yerba II, por lo demás llenos de evidencia de recursos marinos, también contienen caracoles de lomas y, en algunos contextos, en cantidades considerables (figura 7).

En los contextos Mesolíticos de diferentes lugares del mundo, los caracoles han contribuido a cubrir parte de las necesidades diarias de calorías y proteínas (p.ej. Rizner et al. 2005). Por las 
evidencias registradas consideramos que durante el Precerámico Medio los caracoles jugaron un rol importante en la costa sur. Comparados con los moluscos marinos, un caracol proporciona poco alimento, a lo que además hay que sumarle la dificultad de extraer la parte comestible. Sin embargo, se pueden recolectar grandes cantidades de caracoles en el invierno y, a diferencia de los moluscos marinos, pueden conservarse frescos durante meses por su capacidad de "invernar" sellando la concha con una lámina formada de "baba" que ellos producen (Ramírez 1988). La importancia de los caracoles en la dieta precerámica podría establecerse mejor si consideramos que la disponibilidad de los recursos varía con las estaciones, particularmente cuando los efectos de El Niño se hacen sentir en ciertas partes de la vida marina como las machas (Mesodesma sp.), tan evidentes en estos basurales precerámicos, pero intolerantes a cualquier aumento en la temperatura del mar.

La mayoría de las conchas de caracol de tierra están completos y solo muestran pequeñas señales de alteración. Pero, en el caso de los caracoles de Amara Norte I, hemos observado que algunas conchas cuentan con un agujero pequeño, lo cual sugiere que se extrajo el alimento utilizando una espina de cactus (figura 4D). Al considerar que la variedad de especies de moluscos marinos y terrestres en los diferentes contextos son similares -relacionado con otra clase de restos orgánicos- nosotros usamos el número mínimo de individuos (MNI), como un "dato" para medir la importancia relativa que tienen los recursos de las lomas en algunos contextos. El procedimiento descrito nos ofrece una estimación básica para comparar el uso de los recursos de las lomas en los diferentes sitios del periodo Precerámico Medio (figura 6).

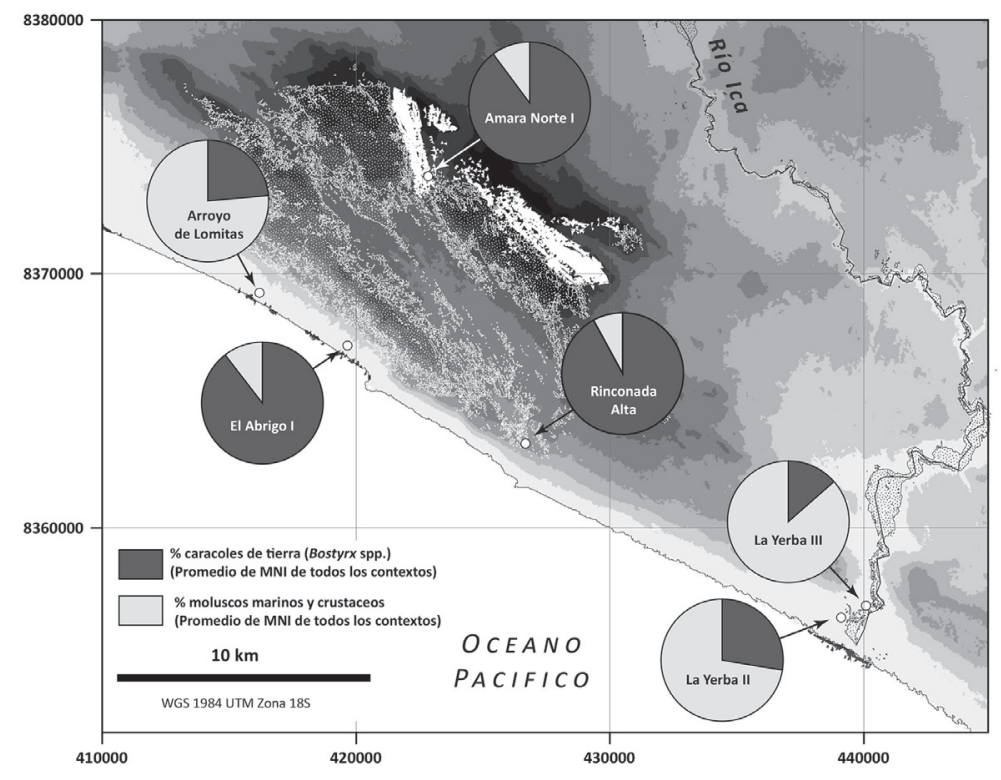

Figura 6. Caracoles terrestres (Bostyrx spp.) como proporción del conjunto total de moluscos y crustáceos (por el número mínimo de individuos 'NMI') en los sitios del Precerámico Medio de la costa sur

Si bien es posible medir la importancia que tuvieron otras especies de animales para los cazadores-recolectores del Precerámico Medio a través de los restos óseos conservados -aunque es importante tener presente que las diferencias en cada sitio pueden responder a los procesos tafonómicos- también es posible conocer el rol de ciertas especies a través del estudio de los instrumentos de piedra utilizados para la caza o procesamiento de las presas. 
Aún viven tropillas de guanacos en las lomas de San Fernando y Amara (figura 6D). Los camélidos silvestres son tímidos y se desplazan por zonas de difícil acceso dentro de las lomas. En el pasado los guanacos, empujados por su necesidad de "pasto" y agua, debieron haberse desplazado hacia las lomas en invierno y hacia el río (y los ecosistemas circundantes) en verano. Esta movilidad debe haber establecido las rutas de trashumancia de los cazadores-recolectores (Custred 1979). Los guanacos junto con el venado de cola gris (Odocoileis virginianus) y predadores como el puma (Puma concolor) fueron más numerosos en las lomas y en los bosques de los valles occidentales de la costa peruana (Wheeler et al. 2011; Beresford-Jones 2011). En estos días los venados de la costa sur solo viven en cautiverio. Sin embargo, los contextos precerámicos cuentan con huesos de venados y camélidos (probablemente guanaco), y otros vestigios asociado con la práctica de la caza en las lomas (Lavallée y Julien 2012).

En el Precerámico Medio aún es desconocida la tecnología del tejido, por esa razón la caza de mamíferos en las lomas también proporcionó piel y tendones, elementos vitales para la elaboración de "ropa" y "cuerdas". Una referencia importante es el caso de los cazadores-recolectores Yámana de Tierra del Fuego, quienes prefirieron los cueros de guanaco para elaborar ropa en vez del cuerpo de mamíferos marinos, ya que la piel del guanaco es más delgada y flexible, lo que permite que se ajuste mejor a los contornos del cuerpo humano. Además, el pelaje del camélido es más grueso, lo cual permite preservar mejor el calor y ser más impermeable (Lothrop 1928). Los contextos de La Yerba II contienen buena evidencia de la preparación de pieles en forma de numerosas herramientas raspadoras hechas de concha de Choromytilus y plantas potencialmente utilizadas para curar cuero (ver abajo).

Dadas las características climáticas de las lomas, la preservación de los restos orgánicos es reducida, a pesar de ello hemos recuperados algunos fragmentos de huesos de ungulados en el sitio Amara Norte I. En La Yerba II el 17\% (de MNI) de los huesos de animales corresponden a camélidos y venados. De estos últimos se tienen los huesos de todo su esqueleto, mientras que los de camélidos, en su mayoría (85\%), corresponden a zonas carnosas como las patas traseras. Esto sugiere que los grandes mamíferos fueron cazados lejos de los sitios y regresaron a ellos solo con las partes más carnosas.

A través de las lomas de la costa sur es posible encontrar obsidiana y otras herramientas de piedra, en su mayoría puntas de proyectil para cazar. También se documentaron pequeñas estructuras de piedra próximas a las rutas de transito que usan hoy las tropillas de guanaco. Un ejemplo de ese tipo de sitio es el Abra Sur de Amara II, el cual interpretamos como un "punto" de observación para la caza (comparar con Larrain et al. 2001). Sitios ubicados en el interior de las lomas y asociados con grandes cantidades de conchas de caracol, como Amara Norte I, están rodeados de lascas y puntas de proyectil de obsidiana que, presumiblemente, fueron usados para cazar (figura 5B).

\section{La flora de las lomas como recurso comestible}

La importancia que tienen los vegetales dentro de la dieta de cazadores-recolectores que no viven en latitudes extremas es reconocida por los investigadores (p.ej. Cordain et al. 2002). En líneas anteriores hemos señalado que la vegetación de las lomas sobrevive a las temporadas de aridez mediante órganos de almacenamiento (geófitos), los cuales pudieron proveer de un invalorable recurso para los cazadores-recolectores del Precerámico Medio (Engel 1987). Los representantes más significativos documentados en las lomas de la costa sur son: raíces tuberosas de Aa weddelliana (Orchidaceae), recientemente documentadas en otro lugar de la costa peruana (Trujillo y Delgado Rodríguez 2011), raíces de Alstroemeria sp. (Alstroemeriaceae), otras especies cercanas a las nombradas que en las lomas de Chile proveen alimentos para humanos y son 
conocidas como “chuño” (Puga 1921; Muñoz-Schick y Moreira-Muñoz 2003); y los bulbos de varias especies de Oxalis (Oxalidaceae) como O. lomana (Stafford 1939 reportó que su sabor es parecido a la avellana; figura 5B). Diversas variedades de papas verdaderas y silvestres se encuentran en la vegetación de lomas en otras partes del Perú (Sandra Knapp, comunicación personal), aunque solo Solanum montanum, una especie muy variable relacionada lejanamente con las papas (Bennett 2008), se registra hoy en las lomas de la costa sur (figura 5C). Como todos los tubérculos de Solanaceae, estos son amargos y deben ser procesados antes de consumirlos. En tiempos históricos estos fueron registrados como un alimento de menor importancia (Hooker 1827) y Dillon (2005:141) informó sobre el cultivo de papas domesticadas (Solanum tuberosum) en las lomas durante los eventos de El Niño. Los geófitos de lomas comestibles son más nutritivos antes y después de los períodos de crecimiento activo, pero permanecen disponibles todo el año y se recolectan fácilmente porque las plantas como Solanum montanum se encuentran en parches concentrados y no están profundamente enraizadas (Cohen 1978; Ochoa 1998).

Semillas comestibles, verduras y frutas de las lomas son un recurso estacional que provee importantes vitaminas y micronutrientes. En particular, todos los cactus de las lomas producen un fruto de sabor agradable y contribuye significativamente a la dieta en diferentes momentos del año. El Corryocactus brachypetalus, que crece únicamente en los farallones rocosos de las lomas que miran hacia el mar, tiene la fruta más grande cuya pulpa tiene un gusto semejante al aguaymanto. Dos especies del cactus Haageocereus (decumbens y aff. Tenuis), que se encuentran generalmente en la zona de transición de vegetación de las lomas de Amara, producen frutas de jugo dulce en el mes de enero, momento en el que la niebla de invierno se ha retirado. Los frutos de Cumulopuntia sphaerica y Eriosyce islayensis son comestibles pero su sabor no es tan agradable.

La buena preservación de los restos orgánicos en los contextos del Precerámico Medio de La Yerba II, ubicado cerca al estuario del río Ica, evidencia el uso y consumo de plantas de lomas: tubérculos de Oxalis spp., y frutos y semillas de cactus (Haageocereus spp., figura 7G). Evidencias indirectas del consumo de plantas son las piedras para moler ("batanes") encontradas en La Yerba II y III (figura 7C), y también en Amara Norte I, ubicado en el corazón de las lomas (figura 4F), donde las plantas se conservan solo en forma de carbón. La presencia del tejido parénquima carbonizado propio de alimentos almidonados está presente en todos los sitios del Precerámico Medio. En La Yerba II se registraron espinas de Haageocereus atadas con cuidado, las cuales probablemente fuero usadas para elaborar anzuelos (Engel 1984).

Hoy en día algunas plantas de las lomas son conocidas por sus propiedades antibacteriales y medicinales, ejemplos de ellas son Ephedra spp. y Plantago spp. (Villagrán y Castro 2003). La Krameria lappacea es utilizada en tratamientos de inflamación, enfermedades gastrointestinales y otros propósitos médicos (Simpson 1989; Brack Egg 1999). La Krameria también tiene un alto contenido de taninos y, en ocasiones, es utilizada para curtir pieles de animales. Restos de ella fueron identificados en contextos precerámicos de La Yerba II (figura 7I). La mayoría de estos contextos arqueológicos también contienen muchos hilos de pesca finamente hilados y fragmentos de redes. Sugerimos que, antes del algodón, estos se hilaban a partir de fibras finas del líber, como las de Asclepias spp., u otra planta estrechamente relacionada de la familia Apocynaceae (Beresford-Jones et al. 2018).

Muchas de estas plantas son importantes en la dieta de los animales que fueron cazados en el Precerámico. Los guanacos, por ejemplo, se alimentan de hierbas leguminosas de las lomas como la Astragalus, además de raíces y tubérculos de plantas perennes, hierbas fruto de cactus. Además, en invierno y verano pueden alimentarse de líquenes y Tillandsia que predominan en la costa del sur (Raedeke y Simonetti 1988; Reus et al. 2009). 

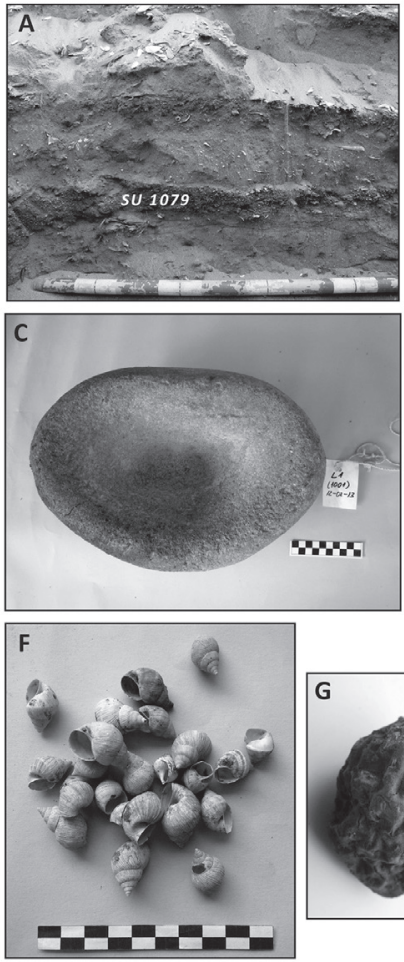
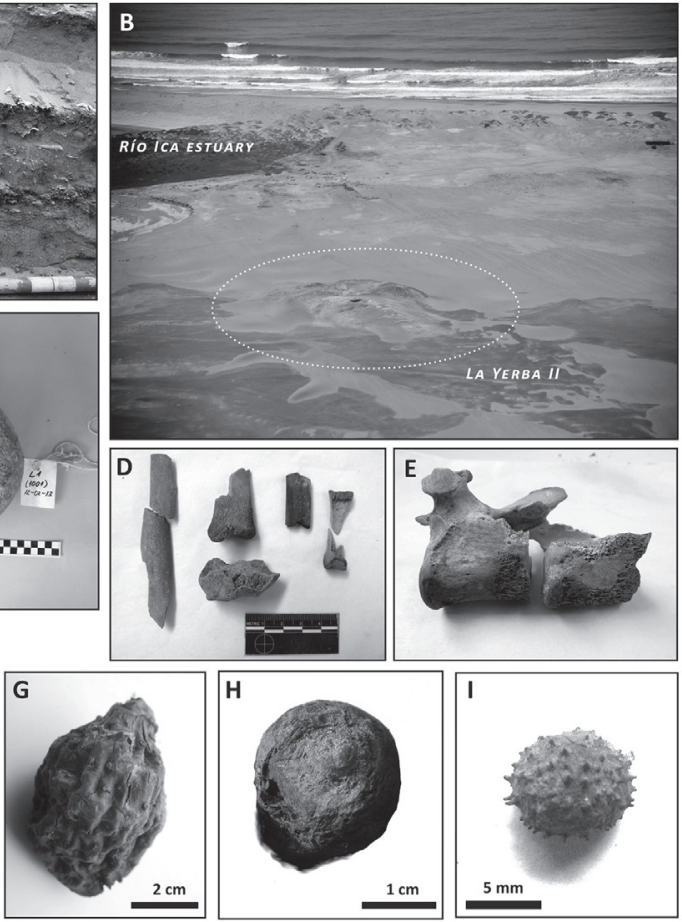

Figura 7. Recursos de las lomas en los contextos precerámicos del sitio La Yerba II, boca del río Ica: A) contexto Precerámico Medio, Trinchera 3, La Yerba II. Unidad estratigráfica SU 1079 fechado 6936-6735 Cal AP; B) vista sur de La Yerba II, 2013; C) mortero de piedra ('batan’), La Yerba II, SU 1001, presumiblemente para procesar plantas alimenticias; D) restos de huesos procesados de venado gris (Odocoileus virginianus), La Yerba II, SU 1001; E) ejemplo de huesos procesados, vertebra de camélido, posible guanaco (Lama guanicoe), La Yerba II, SU 1001; F) conchas de caracol de tierra Bostryx sp., La Yerba II, SU 1005; G) fruta desecada del cactus Haagocereus sp., La Yerba II, SU 1072; H) Fragmento de tubérculo desecado de Oxalis sp., La Yerba II, SU 1079; I) semilla desecada Krameria sp., La Yerba II, SU 1072

\section{La flora de las lomas como combustible}

En todos los sitios precerámicos sus ocupantes utilizaron combustibles para cocinar, abrir las almejas (Waselkov 1987:100), curtir pieles de animales, asar ciertos tubérculos de las lomas y, obviamente, para calentarse porque en los trópicos la costa sur del Perú es notablemente fría en invierno, cuando las temperaturas bajan considerablemente y los fuertes vientos traen nieblas húmedas cerca de la costa del Pacifico. En las lomas de la costa sur la temperatura promedio durante el invierno es de $7^{\circ} \mathrm{C}$.

La evidencia procedente de los sitios precerámicos como La Yerba II y III, ubicados cerca del río, sugiere el uso de especies forestales y del estuario como combustible. En el caso de los sitios ubicados dentro de las lomas, sugerimos que debió utilizarse la vegetación propia del lugar porque los estudios etnográficos indican que los recursos empleados como combustible, como la leña, casi siempre se recolecta dentro de un radio no mayor a dos horas de caminata (Willis y Van Andel 2004:2371). Cantidades considerables de carbón vegetal fueron registrados en el interior de fogatas documentadas en el Arroyo de las Lomitas (figura 4E). Este conjunto de carbón está 
compuesto principalmente por Atriplex rotundifolia (75\%), pero también por carbones de árboles leguminosos (Prosopis o Acacia), sauce (Salix humboldtiana) y varios arbustos leñosos como Baccharis sp., Grabowskia boerhaaviaefolia, Scutia spicata y Tessaria integrifolia y cactus. Aunque la madera arrastrada por el mar podría haber contribuido a la economía general de combustible, hoy en día es escasa a lo largo de esta costa rocosa. En sitios dentro de las lomas como Amara Norte I solo se conservan microrrestos de carbón, pero es posible encontrar piedras tiznadas y termofracturadas como evidencia de continuas fogatas.

En la actualidad, en las lomas de la costa sur no existen árboles y solo se pueden encontrar algunas especies arbustivas como Ephedra americana, A. rotundifolia y C. alnifolius que, como todas las especies leñosas de lomas, son de crecimiento lento, pero productoras de combustible de alto valor calorífico, lo que las hace propensas a ser sobreexplotadas. En otros sitios, el carbón precerámico se ha tomado como evidencia de tal sobreexplotación (Weir y Derring 1986). Los restos de fogatas registrados en el sitio Arroyo de Lomitas sugieren que las lomas proveyeron de muchos arbustos. Además, grandes áreas de las lomas están cubiertas de Tillansdia spp., especie que fue utilizada como combustible en el Precerámico (Moseley y Willey 1973; Weir y Derring, 1986; Quilter 1991). Las fibras suaves de sus semillas hacen una yesca eficiente. Sin embargo, la mayoría de Tillandsia son de crecimiento lento (Craig 1985) y su sobre explotación pudo haber ocasionado desestabilización en los sistemas de dunas (Hesse 2012:35), evidente en sitios como Amara Norte I.

La historia de la ecología humana del Precerámico en las lomas de la costa sur cubre varios milenios, $\mathrm{y}$, sin embargo, es posible ver a través del registro arqueológico los cambios vividos. En la siguiente sección expondremos la evidencia que nos permitirá contribuir a la importante pregunta sobre las causas detrás de tales cambios.

\section{CAMBIOS A TRAVÉS DEL TIEMPO}

Las lomas, como otras zonas ecológicas áridas, son frágiles y particularmente sensibles a las perturbaciones climáticas (Masuda 1985) y el impacto humano (Dillon et al. 2003).

Tal como ha sido discutido, el factor climático de mayor relevancia en las lomas consiste en las periódicas perturbaciones que existen a lo largo de la costa del Perú a causa del fenómeno del ENOS (Oscilación del Sur-El Niño) El ENOS se caracteriza por anomalías en la temperatura de la superficie del mar (TSM), las cuales han sido descritas de acuerdo a dos modos espaciales de variabilidad: las anomalías máximas en la TSM localizadas en el Pacífico central (modo 'PC' o 'Modoki El Niño'), que implican anomalías negativas en la TSM de la costa peruana y fuertes eventos de La Niña; y las anomalías máximas en la TSM localizadas en el Pacífico oriental (modo 'PO'), vinculadas con fuertes eventos de El Niño (e.g. Carré et al. 2014).

La reconstrucción de la historia de ENOS mediante diversas líneas de datos indirectos (o proxy data), incluidos en sedimentos lacustres y marinos, núcleos de hielo y sitios arqueológicos conlleva muchas complejidades, y fueron recientemente revisadas por Sandweiss et al. (2020). Un modelo del ENOS histórico establecido desde hace un tiempo, basado en datos de sitios arqueológicos en la costa norte del Perú (Sandweiss et al. 1996, 2020; Sandweiss y Kelley 2012) sugiere cerca de cuatro milenios de supresión del ENOS durante el Holoceno Temprano, entre los años 9 y 6 ka, después de los cuales ocurrió una baja frecuencia de El Niño hasta el 3 ka, momento en el cual se fue estableciendo el régimen moderno que conocemos como El Niño (Rodbell et al. 1999; Moy et al. 2002). Sin embargo, es evidente que el clima costero y las condiciones oceanográficas demuestran una variación significativa al sur de la latitud $12^{\circ} \mathrm{S}$ durante los eventos ENOS, con efectos más pronunciados que hacia el norte de esta latitud. Estas variaciones incluyen otras variantes del ENOS como el ‘El Niño Costero' que sufrió la costa norte y central en el 2017 (Sandweiss et al. 2020). 
Al sur de $12^{\circ} \mathrm{S}$ se han propuesto variaciones específicas para la costa sur para este modelo, basados en un $\delta^{18} \mathrm{O}$ como indicador (proxy) para la temperatura superficial del mar, registrada en conchas de almejas halladas en depósitos arqueológicos, incluyendo los sitios La Yerba II, los corales del Pacífico central y foraminíferos de las islas Galápagos (Carré et al. 2014). Todo esto sugiere que por más de cinco milenios, entre los años 9,6 y 4,5 ka, las medias anuales de la TSM fueron significativamente más bajas que hoy en día, especialmente al sur del Perú; que antes del 8 ka el ENOS era más como el El Niño (modo 'PO'), mientras que entre los años 7,5 y 6,7 ka el fenómeno se inclinó hacia el modo 'PC' con eventos de La Niña más frecuentes e intensos; y entre los años 5 y 4 ka ocurrió un período de reducción sustancial en la varianza del ENOS. ¿Cómo podría haber impactado entonces este modelo revisado del ENOS en las lomas de la costa sur? ¿Cómo fue tal impacto representado en el registro arqueológico?

Las variaciones en el modo espacial y la amplitud del ENOS afectan las temperaturas relativas de la tierra y el mar que impulsan los regímenes de viento de convección y gobiernan otras interacciones mar-atmósfera, como la producción de aerosoles y núcleos de condensación y, por lo tanto, la producción, la intensidad (tamaños de las gotas de lluvia) y la duración de las precipitaciones ocultas en las lomas. En el norte y centro del Perú, El Niño provoca 'enormes eventos de florecimiento' (Muenchow et al. 2013:564) y un aumento explosivo en la población de caracoles (Ramírez et al. 2003) en las lomas (véase también Cano et al. 1999; Dillon et al. 2003; Kalicki et al. 2014). Más al sur también incrementa la vegetación de las lomas, debido a la niebla de advección que transporta un alto contenido de humedad en aguas más cálidas (Eichler y Londoño 2013:1, véase también Muñoz-Shick et al. 2001, Manrique et al. 2010). Dillon (2011) registró un incremento de hasta 13 veces en la productividad primaria de las lomas al sur del Perú, medido a partir de la cobertura y densidad vegetal, durante el poderoso evento de El Niño de 1997-98.

Los efectos de los eventos de La Niña en las lomas peruanas son menos sencillos de comprender. Tierra adentro, las condiciones oceánicas más frías durante La Niña se traducen en condiciones más secas. Aun así, inmediatamente a lo largo de la costa, La Niña crea neblinas más persistentes (Manrique et al. 2010; Muenchow et al. 2013; Eichler y Londoño 2013), porque las masas de aire húmedo más fríos que transporta son más propensas a la condensación (McPhaden et al. 2006). Muenchow et al. (2013:564) registran "abundantes eventos de florecimiento, una alta diversidad en especies y una alta cobertura en especies", aunque este registro fue durante un solo año de La Niña en Casma. Pese a ello, la relación entre un aumento de la neblina y la vegetación de las lomas está lejos de ser simple, en particular porque está gobernada también por cambios en la altitud y temperatura de la capa de neblina (Manrique et al. 2010; Eichler y Londoño 2013; Muenchow et al. 2013). En consecuencia, las especies de plantas de las lomas han evolucionado en estos regímenes húmedos y nichos topográficamente delimitados para conformar un mosaico complejo de vegetación. Las condiciones persistentes de La Niña pueden, por ejemplo, promover el surgimiento de especies xerófitas y ampliar los cordones de vegetación transicional en perjuicio de las hierbas producidas por las neblinas (Latorre et al. 2011).

Para las lomas de la costa sur, el impacto del ENOS en los recursos de las lomas es bimodal: primero, El Niño tiende a producir una mayor biomasa sobre tierra en zonas efímeras, mientras que La Niña tiende a producir una mayor biomasa subterránea en forma de geófitos en las zonas de niebla (Beresford-Jones et al. 2015b). En resumen, podemos inferir que, en tiempos pasados, hubo una mayor varianza e intensidad de ENOS durante varios siglos -ya sea de La Niña o El Niño- que habrían reforzado la extensión, volumen y la altura de la vegetación atrapanieblas de las lomas, influyendo positivamente en la hidrología de la biomasa y produciendo un microclima autosustentable. Esto habría aumentado de manera importante la biomasa de los recursos de flora y fauna explotados por los cazadores-recolectores del Precerámico. Diferentes modos de ENOS hubieran promovido diferentes partes del ecosistema de las lomas, lo cual habría conllevado diferentes estrategias de subsistencia, por ejemplo, concentrándose en la caza de guanaco y recolección 
de caracoles a medida que sus poblaciones aumentaban debido a la expansión de la vegetación de las lomas durante periodos de gran variación de El Niño, o, concentrándose en recolectar las plantas geófitos -ricas en almidón- de las lomas perennes cuando prosperaban gracias a nieblas más persistentes en épocas de mayor variación de La Niña.

En contraste, largos periodos de supresión en la varianza de ENOS hubieran tenido un impacto negativo sobre los ecosistemas de las lomas. De hecho, sin regeneración a través de eventos periódicos de ENOS, algunas especies de lomas podrían haber desaparecido completamente con el paso del tiempo (Dillon 2011). También se incrementaría su vulnerabilidad al impacto del hombre (cf. Ballester y Grimberg 2018). La vegetación en las lomas actúa, en general, no como consumidora de agua, sino como un factor de producción de agua debido a las muy mejoradas superficies que proporcionan para la condensación de agua de niebla.

Esto es particularmente cierto en el caso de los árboles y los arbustos leñosos más altos que aumentan la condensación de la niebla en las lomas por un factor de hasta seis veces (Ellenberg 1958); de tal modo que su remoción, digamos, para usos de combustible, actúa reduciendo la hidrología de las lomas y la humedad del suelo y, por lo tanto, el crecimiento y germinación de otras plantas y de la biomasa de las lomas en su totalidad (Engel 1973; Walter 1973; Oka 1986, Muenchow et al. 2013).

La figura 8 muestra el registro arqueológico para las lomas de la costa sur y los últimos datos históricos de clima de ENOS para la misma región, tal como fue sintetizado por Carré et al. (2014). Es importante enfatizar que el análisis de Carré y sus colegas no reconstruye eventos individuales de ENOS, sino que muestra variaciones en las frecuencias de ENOS durante períodos de tiempo profundos. Es este impacto acumulativo en la variación del ENOS a lo largo del tiempo que podríamos argumentar tiene relevancia para determinar los cambios en los estilos de vida de los cazadores-recolectores marinos representados por el registro arqueológico del Precerámico Medio de las lomas en la costa sur del Perú. Hacemos cinco observaciones de esta combinación de datos a la luz de la información ecológica que ya fue presentada anteriormente.

En primer lugar, la Figura 8 nos muestra que, en la escala más amplia, todos los sitios arqueológicos precerámicos íntimamente asociados con las lomas de la costa sureña fueron ocupados durante cinco milenios, en los cuales, tal como lo sintetizan Carré et al. (2014:1045), "la media TSM anual fue significantemente más baja...que hoy en día, especialmente al sur del Perú, unos $\sim 3^{\circ} \mathrm{C}$ más fría, [lo cual implica] un incremento en la intensidad de la marejada costera". Fue esto lo que dio forma a las condiciones de una productividad oceánica mayor y de una hidrología aumentada de las lomas y biomasa debido a las nieblas más persistentes, las cuales brindaron sustento a los cazadores-recolectores en lo que Engel (1981:24) llama el “fog oasis situation”. Además, el sitio Abrigo I del periodo Precerámico Temprano (10200-9540 años cal AP, tabla 1) nos permite resaltar otro factor que determina la distribución de los sitios antes de la relevancia de las lomas: el nivel del mar (Noller 1993:11; Dillon 2011). En aquellos tiempos el nivel eustático del mar fue 35 m más bajo que en la actualidad. El cambio del nivel ocasionó alteraciones significativas en el relieve costeño y cubrió muchos sitios arqueológicos del Precerámico Temprano (ver Richardson 1998; Sandweiss 2009). Los cambios en el mar también afectaron la distribución de las lomas, ya que ellas están determinadas principalmente por la altitud.

En segundo lugar, hubo, al parecer, un milenio en el cual El Niño era más prevalente (modo PC) antes del 8 ka y al comienzo del período Precerámico Medio, que habría expandido la superficie de vegetación y biomasa de las lomas, pero a la vez, también habría causado choques persistentes en ciertos recursos marinos relevantes para la subsistencia. Solo un sitio junto al estuario, La Yerba I, fue fechado por Engel (1991:54) en esta época, y una calibración posterior implica un margen de incertidumbre de casi un milenio (9018-7693 años cal AP, tabla 1). Engel brinda pocos detalles y nuestras propias investigaciones del mismo sitio -en la medida en se puede determinar- sugieren que tanto la fecha como el contexto arqueológico son, de hecho, similares al sitio adyacente de 
OxCal v 4.4.4 Bronk Ramsey (2021); r:5 SHCal20 atmospheric curve (Hogg et al. 2020)

Abrigo I (I-7772, Engel 1991:56)

La Yerba I (Birm-511, Engel 1991:56)

La Yerba I 2013 SU 1024 (OxA-30913)

La Yerba II (I-7773 Engel 1991:56)

La Yerba II 2013 SU 1015 (OxA-29975)

La Yerba II 2013 SU 1079 (OxA-29944)

La Yerba II (OS-60556, Carré 2014 ICA-IN)

El Campo (I-3560, Engel 1991:56)

Bahia de San Nicolas (Engel 1981:59)

La Yerba III (Birm-513, Engel 1991:56)

La Yerba III (OxA-32290)

La Yerba III (OxA-32291)

Santa Ana (Engel 1981: 61)

Amara Norte I (OxA-32350)

Arroyo de Lomitas (I-7774, Engel 1991:56)

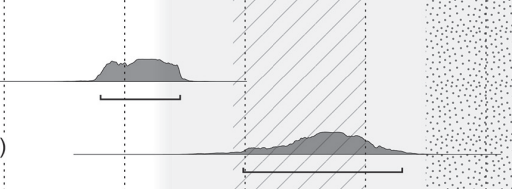

)


La Yerba II (figura 8). De ser así, hay poca evidencia arqueológica visible de ocupación humana de la costa sur durante este periodo, aunque eso también pueden ser el resultado de factores de un cambio eustático y estabilización del nivel del mar, pertinentes a nuestra próxima observación.

En tercer lugar, dentro del extenso rango de cinco milenios de las aguas frías, el comienzo del período Precerámico Medio está marcado por el descubrimiento de diversos sitios arqueológicos de alta visibilidad, el más notable de estos es La Yerba II (7572-6567 años cal AP, tabla 1), situado en el estuario del río Ica. Esto coincide con un período de numerosos siglos adonde se ve incrementada la actividad de La Niña (figura 8), lo que resulta en mares aún más fríos y lomas con mayor presencia de niebla: condiciones reflejadas en la ecología, particularmente de aguas frías, en partes de la colección de moluscos de La Yerba II, tales como Tegula atra y Choromytilus chorus. Este momento es también cuando se estabilizó el nivel eustático del mar, con lo cual se formaron las playas arenosas que favorecieron el crecimiento y reproducción de grandes bancos de machas, recurso marino de alta frecuencia en los conchales de La Yerba II. Por lo tanto, no es casualidad que el establecimiento de la forma de vida propia del Precerámico Medio haya tomado lugar en una época donde los recursos oceánicos y el de las lomas fueron abundantes y predecibles.

En cuarto lugar, en los cerca de quinientos años que bordean el $6 \mathrm{ka}$, los sitios arqueológicos en los estuarios de los ríos muestran evidencia de un incremento del sedentarismo y un crecimiento de la base de recursos en un espectro de subsistencia mixto de caza y cultivo (comparar con Gorbahn 2020). Mientras que sitios como La Yerba II se caracterizan por refugios contra el viento hechos de junco (comparar con Quilter 1989); sitios fechados posteriormente junto a los estuarios del río, como La Yerba III y Santa Ana (6493-5895 años cal AP y 5656-4980 años cal AP, respectivamente, tabla 1) contienen evidencia de aldeas permanentes con una disposición mortuoria estructurada (Engel 1981:20-21, 1991:157, comparar con la 'fase Encanto' en otras citas; Lanning 1963; Patterson y Moseley 1968). Estos últimos sitios tienen grandes cantidades de obsidiana, lo cual indica unas esferas de interacción mucho más amplias ya que las fuentes más cercanas están a 250 km, en las tierras altas de la zona de Huanca Sancos en Ayacucho (Tripcevich y Contreras 2011). Esos sitios también tienen la primera evidencia local del desarrollo de una agricultura de alimentos cultivada en los cienos húmedos de las adyacentes llanuras aluviales de los ríos. En este sentido, recuperamos pallares completamente domesticados (Phaseolus lunatus), mientras que Engel (1981: 20) reportó frejoles Phaseolus y jícama (Pachyrhizus tuberosus) en los contextos de La Yerba III.

Por último, pero no menos importante, la figura 8 sugiere que, a medida que la larga época de temperaturas marinas más frías terminaba alrededor de 4,5 ka y en sincronía con un Holoceno con un mínimo de varianzas ENOS que duró mil años hasta 4 ka, cada uno perjudicial al ecosistema de lomas y las fuentes de aguas que alimentaban a lo largo del litoral, también llego a su fin una forma de vida del Precerámico Medio que había existido aquí por milenios alrededor de las estaciones de las lomas y su rico litoral oceánico.

En efecto, la significancia de los recursos de las lomas para el asentamiento humano está clara por el hecho de que no hay sitios arqueológicos precerámicos a lo largo de la costa entre Morro Quemado y Bahía de San Nicolás fechados después del año 4450 años cal AP. La fecha aceptada para el final del período Precerámico Medio en cualquier otra parte del Perú es 4450 años AP sin calibrar (Quilter 1991). Calibrado usando ShCal20 (Hogg et al. 2020), esto se ubica en aproximadamente 5000 años Cal AP. Parece haber pocos motivos para suponer que un giro hacia temperaturas marítimas promedio, afines a las condiciones modernas, junto con una actividad suprimida del ENOS, habría sido perjudicial para muchos recursos marinos, en particular, para las machas (Mesodesma sp.) las cuales por largo tiempo fueron un componente dietético clave. $\mathrm{Y}$, aun así, los únicos sitios en el área de estudio que datan en el subsiguiente periodo del Precerámico Tardío son pequeños campamentos logísticos como Amara Norte o Arroyo de Lomitas. ${ }^{2}$ Aparentemente, faltan en el registro arqueológico Precerámico Tardío, los sitios más grandes en 
los estuarios de río, como el campamento base de La Yerba II o los poblados permanentes como La Yerba III o Santa Ana. Continuando con el colapso de la "fog oasis situation", por lo tanto, especulamos que un aumento en la dependencia de agricultura durante el Precerámico Tardío ameritó un traslado de los asentamientos tierra adentro, en las cuencas ribereñas de los ríos costeros del sur del Perú.

Por otro lado, en las costas norte y centro del Perú este cambio crítico llevó al crecimiento de la densidad poblacional y la aparición de arquitectura monumental después de los 5000 años cal AP (Shady y Leyva 2003; Alva 2014; Dillehay 2017; Prieto y Sandweiss 2020). Esto no sucedió en la costa sur, probablemente por su configuración geomorfológica distintiva. A diferencia de los valles ribereños al norte, con sus extensas deltas aluviales y tierras frente al mar que garantizan un fácil acceso a recursos marinos y agrícolas, los sistemas fluviales de la costa sur están compuestos de cuencas ribereñas repartidas por los largos cursos de río, desviados y separados del mar por las mismas formaciones costeñas de las lomas que fueron el primer anfiteatro de la ecología humana durante el Precerámico Medio.

En tiempos arqueológicos tardíos, incluso en tiempos históricos, las lomas continuaron siendo visitadas para recolectar caracoles, plantas, hierbas para animales domesticados e, incluso, realizar cacerías de animales (Rostworowski 1981; Masuda 1985; Larrain et al. 2001; Beresford-Jones 2011; Kalicki et al. 2014).

Los caminos a través de las lomas de la costa sur eran aún recorridos para acceder a recursos marinos a lo largo del litoral (Engel 1991). Aunque parece claro que, para esos tiempos posteriores, los recursos en las lomas y en el mar fueron estrictamente suplementarios (Carmichael et al. 2014) y nunca volvieron a dictar los caminos de la existencia humana tal como lo hicieron durante el período Precerámico Medio.

\section{CONCLUSIONES}

Es obvio que el mar frio y sus abundantes recursos fueron una fuente inagotable de proteínas para los cazadores-recolectores del periodo Precerámico Medio en la costa del Perú. Sin embargo, y debido a que los recursos marinos se encontraban ubicados en puntos clave a lo largo del litoral, las lomas y sus fuentes de agua dulce fueron los que definieron la configuración de la ecología humana en el Precerámico y, por tanto, el patrón de ocupación y rutas de desplazamiento a lo largo de la costa sur entre Morro Quemado y Bahía de San Nicolás: la "fog oasis situation" de Engel (Engel 1981:24; figura 1). En este escenario, las lomas de la costa sur ofrecen las mejores condiciones para conocer como los cazadores-recolectores explotaron sus recursos.

La flora y fauna de las lomas siempre fueron importantes componentes de la dieta del Precerámico (figura 7) incluso en temporadas críticas, en particular los tubérculos, ungulados y caracoles de lomas. Además, las lomas proveyeron de combustible, medicina y materia prima. Nuestros datos refutan las opiniones que aseguran que las lomas no fueron importantes para el hombre, o que no se habrían alterado durante el Holoceno. Por el contrario, en las lomas se encuentran los parientes silvestres de la papa andina, el tomate (Solanum spp.) y la papaya (Carica sp.), así como el guanaco, pariente silvestre de la llama. Por lo antes dicho, sugerimos que debería reevaluarse el rol que jugaron las lomas en el proceso de domesticación, especialmente tomando en consideración que toda vez que se ha observado la domesticación de camélidos y tubérculos a menudo se han desarrollado en conjunto (Pearsall 2008:113).

El último modelo sobre el comportamiento histórico de los ENOS basados en el registro del isotopo $\delta^{18} \mathrm{O}$ (Carré et al. 2014), junto con los patrones arqueológicos documentados aquí y fechados con gran precisión cronológica, muestran la estrecha relación entre la ocupación del Precerámico Medio de las lomas de la costa sur y una larga época de un mar significativamente 
más frío, con implicancias de mayor intensidad de afloramientos de agua marinas y mayor persistencia de nieblas en las lomas. Los asentamientos humanos incluyen campamentos logísticos dispersos entre el litoral de las lomas y próximos a arroyos alimentados por las nieblas, y sitios emplazados en el interior de las lomas que sirvieron para cazar y recolectar.

Dentro de esos cinco milenios de mar frio, las primeras ocupaciones del Precerámico Medio fueron establecidas cerca a los estuarios durante un periodo multicentenario de mayor actividad de La Niña, lo que implico aún más abundantes recursos oceánicos y de lomas, coincidiendo también con el momento en que los niveles eustáticos del mar se estabilizaron y la progradación de la costa comenzó a formar el hábitat de playa necesario para la abundante y fácil recolección de las macha que dominan los basurales de estos sitios. Estos campamentos logísticos en el estuario del río ofrecieron a los cazadores-recolectores acceso a un mosaico complejo de entornos diversos y altamente productivos.

Eventualmente, los cinco milenios del periodo del Precerámico Medio definidos por la "fog oasis situation" y la época de mares fríos culminó, dejando el paso a un Holoceno con ENOS cuyas características perjudicaron a las lomas y las fuentes de agua que lo sostenían. Este reciente dato sobre las lomas de la costa sur comprueba la perspicaz opinión de Lanning (1963:369), quien afirmó hace medio siglo que el cambio climático -específicamente ligado a las alteraciones de la circulación oceánica (por ejemplo, ENOS) - afectaría de manera significativa el potencial de los recursos de las lomas durante el Holoceno y, por tanto, explicaría porque la ecología humana cambio luego de la "fog oasis situation".

Sin embargo, aunque la propuesta de considerar que el cambio climático afectó la ecología humana del Precerámico Medio es convincente, este no excluye al factor humano de la explicación de la culminación de "fog oasis situation". La actividad humana pudo haber hecho más sensibles a las lomas frente a los cambios climáticos. La acción humana negativa sobre la vegetación de las lomas y, específicamente, sobre los arbustos leñosos - de crecimiento lento y fácil sobre explotaciónque actúan como condensadores de las nieblas pudo profundizar los efectos de cambio climático y éste, a su vez, actuar contra el crecimiento de la vegetación. El registro de carbón vegetal en los fogones del Precerámico Medio en las lomas nos dice que la actual ausencia de especies de árboles y arbustos leñosos es relativamente reciente. Más importante aún, este modelo de declive climáticamente inducido de recursos de lomas hacia el final del Precerámico Medio no parece explicar el surgimiento de la agricultura en los Andes, como Lanning (1967) y otros propusieron.

Los sitios La Yerba II y III fueron ocupados durante más de un milenio hasta el 6000 AP y en sus contextos arqueológicos hemos documentado evidencia relacionada con los cambios que acompañan la aparición de la agricultura en muchas partes del mundo. Para referirse a esta etapa de cambios, Flannery (1969) acuñó el término "Broad Spectrum Revolution". Los cambios que hemos registrado incluyen arquitectura permanente, tumbas -posibles marcadores de territorialidad-, redes de intercambio muy extensas y dinámicas (gran cantidad de obsidiana) y uso creciente de recursos cultivados en las llanuras aluviales como los frejoles, de alto valor proteico, y, finalmente, algodón, el cual permitió la intensificación de la explotación de recursos marinos (Moseley 1975; Beresford-Jones et al. 2018). No obstante, durante esta época de cambio, la ecología humana propia de "fog oasis situation" prevaleció (figura 8).

Por lo tanto, en el caso de la costa sur del Perú el "Broad Spectrum Revolution" se desarrolló, no por la presión demográfica en un contexto de ambientes sobre explotados (Patterson y Moseley 1968), sino, al contrario, como resultado de la abundancia de recursos que la "fog oasis situation" brindó durante todo el Precerámico Medio. Situación que ha sido observada en muchas partes del mundo (Arnold 1996:98; Zeder 2012:258), donde una combinación entre abundancia y predictibilidad de la estacionalidad de los recursos hizo que los cazadores-recolectores -cada vez más complejos- del Precerámico Medio redujeran su movilidad para instalarse en puntos estratégicos en las zonas de confluencia de múltiples ecosistemas, como los estuarios de los ríos. 
Para referirse al periodo cultural de nuestro interés Engel (1987) utilizaba el término 'Mesolítico'. La arqueología peruana ha dejado de usar este tipo de términos para evitar que las comparaciones con el Viejo Mundo oscurezcan los aspectos particulares de la trayectoria cultural Andina. Nosotros, por el contrario, estamos a favor de utilizar el término en el contexto andino con el objetivo de hacer referencia a las conductas humanas que conjuga el "Broad Spectrum Revolution" en otros partes del mundo. Esto, además, coadyuvaría a resaltar la importancia del caso andino, como uno de los pocos lugares donde se originó de manera independiente la agricultura y la civilización.

\section{AGRADECIMIENTOS}

Agradecemos al Ministerio de Cultura del Perú por otorgar el permiso para el trabajo de campo arqueológico (Resolución Directoral No 933-2012-DGPC-VMPCIC / MC, 19 de diciembre de 2012 y No 386-2014-DGPA-VMPCIC / MC, 22 de agosto de 2014); Don Alberto Benavides Ganoza y la gente de Samaca por facilitar el trabajo de campo; el Leverhulme Trust, UK y el Don Alberto Benavides de la Quintana para financiar el Proyecto Arqueológico One River de la Universidad de Cambridge. Este trabajo se basa en una traducción y actualización de un artículo publicado previamente en Quaternary Science Reviews (Beresford-Jones et al. 2015b).

\section{NOTAS}

1 Corresponde a los $250 \mathrm{~km}$ de la costa del Pacifico que abarca los valles del río Pisco, Ica, Río Grande de Nazca y Acarí. Estos valles comparten una particular geomorfología, clima e hidrología que los hace diferentes a la costa central y norte del Perú (Beresford-Jones 2011) y a la costa que se extiende en dirección a la frontera de Chile (el extremo sur). Por tanto, gran parte de su prehistoria tiene una trayectoria compartida y distintiva, comúnmente etiquetada como "sur" por los arqueólogos (Willey 1971:78).

2 Los sitios más grandes del Precerámico Tardío que conocemos se ubican al norte de las lomas de la costa sur, en Otuma y en la Península de Paracas, ambos notablemente asociados con contextos de lagunas de agua superficial y supuestamente más cálida (Engel 1991).

\section{REFERENCIAS BIBLIOGRÁFICAS}

Alva, I. (2014). Ventarrón y Collud. Origen y auge de la civilización en la costa norte del Perú. Lima: Ministerio de Cultura.

Arnold, J. E. (1996). The Archaeology of Complex Hunter-Gatherers. Journal of Archaeological Method and Theory 3: 77-126.

Ballester, B. y D. Grimberg (2018). Historia de la vegetación y ocupación humana en la costa del desierto de Atacama (Antofagasta, Chile). Hombre y Desierto 22: 143-172.

Benfer, R. A. (2005). Frederic-Andre Engel (1908-2002). Andean Past 7: 1-14.

Bennett, J. R. (2008). Revision of Solanum Section Regmandra (Solanaceae). Edinburgh Journal of Botany 65 (1): 69-112.

Beresford-Jones, D. G. (2011). Lost Woodlands of the Ancient Nasca. Oxford University Press, Oxford.

Beresford-Jones, D. G., S. Arce, A. G. Pullen, G. E. Chauca (2015a). Proyecto de investigación arqueológica 
Samaca. Informe de los trabajos realizados durante la temporada 2014. Presentado al Ministerio de Cultura Lima Junio 2015. Lima, Perú. Ms.

Beresford-Jones, D.G., E. Pomeroy, C. Alday, R. A. Benfer, J. Quilter, T. O'Connell y E. Lightfoot (2021). Diet and Lifestyle in the First Villages of the Middle Preceramic: Insights from Stable Isotope and Osteological Analyses of Human Remains from Paloma, Chilca I, La Yerba III and Morro I. Latin American Antiquity 32(2): 1-19. DOI:10.1017/laq.2021.24

Beresford-Jones, D. G., A. G. Pullen, G. Chauca, L. Cadwallader, M. García, I. Salvatierra, O. Q. Whaley, V. Vásquez, S. Arce, K. Lane y C. French (2018). Refining the Maritime Foundations of Andean Civilization: How plant fiber technology drove social complexity during the Preceramic Period. Journal of Archaeological Method and Theory 25: 393-425.

Beresford-Jones, D. G., A. G. Pullen, O. Q. Whaley, J. Moat, G. E. Chauca, L. Cadwallader, S. Arce, A. Orellana, C. Alarcón, M. Gorriti, P. K. Maita, F. C. Sturt, A. Dupeyron, O. Huaman, K. J. Lane y C. I. French (2015b). Re-evaluating the Resource Potential of Lomas Fog Oasis Environments for Preceramic HunterGatherers under Past ENSO Modes on the South Coast of Peru. Quaternary Science Reviews 129: 196-215.

Binford, L. R. (1980). Willow Smoke and Dogs Tails: Hunter-Gatherer Settlement Systems and Archaeological Site Formation. American Antiquity 45: 4-20.

Brack Egg, A. (1999). Diccionario Enciclopédico De Plantas Útiles Del Perú. Centro de Estudios Regionales Andinos, Cusco.

Bronk Ramsey, C. (2009). Bayesian Analysis of Radiocarbon Dates. Radiocarbon 51: 337-360.

Butzer, K. W. (1982). Archaeology as Human Ecology: Method and Theory for a Contextual Approach. Cambridge: Cambridge University Press.

Cano, A., J. Roque, M. Arakaki, C. Arana, M. La Torre, N. Llerena y N. Refulio (1999). Diversidad florística de las lomas de Lachay (Lima) durante el evento “El Niño 1997-98”. Revista Peruana de Biología 6: 125-132.

Carmichael, P., B. V. Kennedy y L. Cadwallader (2014). Coastal but not littoral: Marine resources in the Nasca diet. Ñawpa Pacha: Journal of Andean Archaeology 34: 1-24.

Carré, M., J. P. Sachs, S. Purca, A. J. Schauer, P. Braconnot, R. A. Falcon, M. Julien y D. Lavallee (2014). Holocene history of ENSO variance and asymmetry in the eastern tropical Pacific. Science 345: 1045-1048.

Cohen, M. N. (1978). Archaeological plant remains from the central coast. Nawpa Pacha: Journal of Andean Archaeology 16: 23-50.

Cordain, L., S. B. Eaton, J. Brand Miller, N. Mann y K. Hill (2002). The paradoxical nature of huntergatherer diets: meat-based, yet non-atherogenic. European Journal of Clinical Nutrition 56, S42-S52. DOI: 10.1038/sj.ejcn. 1601353

Craig, A. K. (1992). Archaeological Occurences of Andean Landsnails. Andean Past 3 127-135.

Craig, A. K. (1985). Cis-Andean Environmental Transects: Late Quaternary Ecology of Northern and Southern Peru. En: S. Masuda, I. Shimada, y C. Morris (eds.), Andean Ecology and Civilization. An Interdisciplinary Perspective on Andean Ecological Complementarity: 23-44. Tokyo, University of Tokyo Press.

Craig, A. K. y N.P. Psuty (1968). The Paracas. Papers, 1(2). Occasional Publication No.1, Dept. of Geography, Florida Atlantic University, Boca Raton. 
DAVID G. BERESFORD-JONES Y OTROS - REVISITANDO LA HIPÓTESIS DE FREDERIC ENGEL: CÓMO LAS LOMAS ...

Custred, G. (1979). Hunting technologies in Andean culture. Journal de la Société des Américanistes 66: 7-19.

Dillehay, T. D. (ed.) (2017). Where the Land Meets the Sea: Fourteen Millennia of Human History at Huaca Prieta, Peru. Austin, University of Texas Press.

Dillon, M. O. (2005). The Solanaceae of the Lomas formations of coastal Peru and Chile. Monographs in Systematic Botany 104: 131-156.

Dillon, M. O. (2011). Floristic Checklist of the Peruvian Lomas Formations. Arnaldoa 18: 7-32.

Dillon, M. O., M. Nakazawa y S. L. Gonzáles (2003). The Lomas Formations of Coastal Peru: Composition and Biogeographic Origin. En J. Haas y M. O. Dillon (eds.), El Niño in Peru: Biology and Culture Over 10,000 Years: 1-9. Fieldiana (Botany). Chicago, Field Museum of Natural History.

Dollfus, O. (1968). Le Pérou. Travaux et Mémoires de l'Institut des Hautes Etudes de l'Amérique Latine. Paris: Université de Paris.

Eichler, T. P. y A. C. Londoño (2013). ENSO Impacts on Lomas Formation in South Coastal Peru: Implications for the Pliocene? Advances in Meteorology 2013: 1-7.

Ellenberg, H. (1958). Nebeloasen in der Kustenwuste Perus. Bonn: Universitatsven.

Engel, F. (1973). New Facts About Pre-Columbian Life in the Andean Lomas. Current Anthropology 14: 271-280.

Engel, F. (1981). Prehistoric Andean Ecology: Man, Settlement and Environment in the Andes. Nueva York, Volume 2: The Deep South. Humanities Press, Hunter College.

Engel, F. (1984). Prehistoric Andean Ecology. Man, Settlement and Environment in the Andes. Vol. Stone Typology, 3. New York, Humanities Press, Hunter College.

Engel, F. (1987). De las Begonias al Maíz. Lima, La Universidad Nacional Agraria Del Perú.

Engel, F. (1991). Un Desierto en Tiempos Prehispánicos. Lima, La Universidad Nacional Agraria Del Perú.

Erlandson, J. M. (2001). The Archaeology of Aquatic Adaptations: Paradigms for a New Millennium. Journal of Archaeological Research 9: 287-349.

Flannery, K. V. (1969). Origins and ecological effects of early domestication in Iran and the Near East. En P. J. Ucko, y G. W. Dimbleby (eds.), The Domestication and Exploitation of Plants and Animals: 73-100. Londres, Duckworth.

Gorbahn, H. (2020). Penil Alto. An Agricultural Village of the Middle Archaic Period in Southern Peru. Forschungen zur Archäologie Außereuropäischer Kulturen Band 17. Harrassowitz, Wiesbaden.

Hesse, R. (2012). Spatial distribution of and topographic controls on Tillandsia fog vegetation in coastal southern Peru: Remote sensing and modelling. Journal of Arid Environments 78: 33-40.

Hesse, R. (2014). Three-dimensional vegetation structure of Tillandsia latifolia on a coppice dune. Journal of Arid Environments 109: 23-30.

Hogg, A. G., T. Heaton, Q. Hua, J. Palmer, C. S. M. Turney, J. Southon, A. Bayliss, P. Blackwell, G. Boswijk, C. Bronk Ramsey, F. Petchey, P. J. Reimer, R. W. Reimer y L. Wacker (2020). SHCal20 Southern Hemisphere calibration, 0-55,000 years cal BP. Radiocarbon 62: 759-778. 
Relaciones de la Sociedad Argentina de Antropología 46 (2), julio-diciembre 2021: 383-413

Hooker, W. J. (1827). Witheringia montana. Mountain Witheringia or St. Lorenzo potato. Botanical Magazine 54(1): 2768.

Isla, J. C. (1990). La Esmeralda: Una ocupación del Periodo Arcaico en Cahuachi, Nasca. Gaceta Arqueológica Andina 6: 67-80.

Kalicki, P., T. Kalicki y P. Kittel (2014). The influence of El Niño on settlement patterns in Lomas de Lachay, central coast, Peru. Interdisciplinaria Archaeologica Natural Sciences in Archaeology Volume V. Issue 2.

Klemm, O., R. S. Schemenauer, A. Lummerich, P. Cereceda, V. Marzol, D. Corell, J. Heerden, D. Reinhard, T. Gherezghiher, J. Olivier, P. Osses, J. Sarsour, E. Frost, M. J. Estrela, J. A. Valiente, G. M. Fessehaye (2012). Fog as a Fresh-Water Resource: Overview and Perspectives. AMBIO 41: 221-234.

Lanning, E. P. (1963). A Pre-Agricultural Occupation on the Central Coast of Peru. American Antiquity 28(3): 360-371.

Lanning, E. P. (1967). Peru before the Incas. Prentice Hall, Englewood Cliffs, N.J.

Larrain, H., E. Sagredob, L. Pérez, B. A. Gonzálezc, P. Cerecedad, P. Osses, F. Velásquez, P. Lázaro y M. Navarro (2001). New evidence of human habitation and hunting activity at the fog oasis of Alto Patache, south of Iquique, Chile. En: Proceedings of the 2nd International Conference on Fog and Fog Collection. Saint John's Canada.

Latorre, C., A. L. González, J. Quade, J. M. Fariña, R. Pinto y P. A. Marquet (2011). Establishment and formation of fog-dependent Tillandsia landbeckii dunes in the Atacama Desert: Evidence from radiocarbon and stable isotopes. Journal of Geophysical Research 116.

Lavallée, D. y M. Julien (eds.) (2012). Prehistoria de la costa extremo-sur del Perú: los pescadores arcaicos de la Quebrada de los Burros (1000-7000 a.P.). Lima, Instituto Francés de Estudios Andinos.

Lothrop, S. K. (1928). The Indians of Tierra Del Fuego. Vol. Contributions from the Museum of the American Indian Heye Foundation. Lancaster, PA: Lancaster Press, INC.

Lynch, T. F. (1971). Preceramic Transhumance in the Callejon de Huaylas, Peru. American Antiquity 36: 139-148.

Mächtle, B. y B. Eitel (2012). Fragile landscapes, fragile civilizations: How climate determined societies in the pre-Columbian south Peruvian Andes. Catena 103: 62-73.

Manrique, R., C. Ferrari, C. y G. Pezzi (2010). The influence of El Niño Southern Oscillation (ENSO) on fog oases along the Peruvian and Chilean coastal deserts. En: Fifth International Conference on Fog and Fog Collection and Dew, Climatology Working Group. pp. 30.

Masuda, S. (1985). Algae Collectors and Lomas. En: S. Masuda, I. Shimada, y C. Morris (eds.), Andean Ecology and Civilization. An interdisciplinary perspective on Andean ecological complementarity: 233-250. Tokyo, University of Tokyo Press.

McPhaden, M. J., S. E. Zebiak y M. H. Glantz (2006). ENSO as an Integrating Concept in Earth Science. Science 314: 1740-1745.

Moseley, M. E. (1975). The Maritime Foundations of Andean Civilization. California, Cummings Publishing Company, Menlo Park.

Moseley, M. E. y G. R. Willey (1973). Aspero, Peru: A Re-examination of the Site and Its Implications. American Antiquity 38: 452. 
DAVID G. BERESFORD-JONES Y OTROS - REVISITANDO LA HIPÓTESIS DE FREDERIC ENGEL: CÓMO LAS LOMAS ...

Moy, M., G. O. Seltzer, D. T. Rodbell y D. M. Anderson (2002). Variability of El Niño/Southern Oscillation activity at millennial timescales during the Holocene epoch. Nature 420: 159-162.

Muenchow, J., A. Bräuning, E. F. Rodríguez y H. von Wehrden (2013). Predictive Mapping of Species Richness and Plant Species' Distributions of a Peruvian Fog Oasis Along an Altitudinal Gradient. Biotropica 45: 557-566.

Muñoz-Schick, M. y A. Moreira-Muñoz (2003). Alstroemerias de Chile. Santiago, Taller La Era.

Muñoz-Schick, M., Z. R. Pinto, A. Mesa y A. Moreira (2001). Fog oases during the El Niño Southern Oscillation (ENSO) 1997-1998, in the coastal hills south of Iquique, Tarapacá region, Chile. Revista Chilena de Historia Natural 74 (2): 389-405.

Noller, J. (1993). Late Cenozoic stratigraphy and soil geomorphology of the Peruvian Desert, $3^{\circ}-18^{\circ} \mathrm{S}$ : A long-term record of hyperaridity and El Niño. Tesis doctoral inédita, Universidad de Colorado en Boulder, Estados Unidos.

Ochoa, C. M. (1998). Ecogeography and Breeding Potential of the Wild Peruvian Tuber-Bearing Species of Solanum. Economic Botany 52: 3-6.

Oka, S. (1986). On trial measurements of the moisture in fog on Loma Ancon - In relation to an investigation into the conditions required for development of Lomas communities. En M. Ono (eds.), Taxonomic and Ecological Studies on the Lomas Vegetation in the Pacific Coast of Peru: 41-51. Tokyo, Tokyo Metropolitan University Makino Herbarium.

Oka, S. y M. Ogawa (1984). The distribution of lomas vegetation and its climatic environments along the Pacific coast of Peru, Geographical reports of Tokyo Metropolitan University. Tokyo, Tokyo Metropolitan University Department of Geography.

Ono, M. (1986). Definition, classification and taxonomic significance of the lomas vegetation. En M. Ono (ed.), Taxonomic and Ecological Studies on the Lomas Vegetation in the Pacific Coast of Peru: 5-14. Tokyo, Tokyo Metropolitan University Makino Herbarium.

Palomino, V. (2006). Report only relict population of the Peruvian guanaco (Lama guanicoe) left in the coastal desert of Las Lomas de San Fernando, Ica 2003. Trabajo presentado en Fortalecer el futuro de los Camélidos: Una oportunidad para crecer. IV Congreso Mundial sobre Camélidos, Santa María, Catamarca, Argentina.

Parsons, M. H. (1970). Preceramic Subsistence on the Peruvian coast. American Antiquity 35(3): 292-304.

Patterson, T. C. y M. E. Moseley (1968). Late Preceramic and Early Ceramic Cultures of the Central Coast of Peru. Nawpa Pacha: Journal of Andean Archaeology 6: 115-133.

Pearsall, D. M. (2008). Plant Domestication and the Shift to Agriculture in the Andes" En: H. Silverman, H. y W. H. Isbell (eds.), Handbook of South American Archaeology: 105-120. Nueva York, Springer.

Philippi, R.A. (1851). Centuria quarta Testaceorum novorum. Zeitschrift für Malakozoologie 8(1): 30.

Prieto, G., y D. H. Sandweiss (eds.) (2020). Maritime Communities of the Ancient Andes. Gainesville, University Press of Florida.

Prohaska, F. J. (1973). New evidence on the climatic controls along the Peruvian coast. En D. H. K. Amiran, y A. W. Wilson (eds.), Coastal Deserts: Their Natural and Human Environments: 91-107. Tucson, University of Arizona Press. 
Relaciones de la Sociedad Argentina de Antropología 46 (2), julio-diciembre 2021: 383-413

Puga, F. (1921). El chuño del ligtu. Revista Chilena de Historia Natural 25: 313-320.

Quilter, J. (1989). Life and Death at Paloma, Society and Mortuary Practices in a Preceramic Peruvian Village. Iowa City, University of Iowa Press.

Quilter, J. (1991). Late Preceramic Peru. Journal of World Prehistory 5: 387-438.

Raedeke, K. J. y J. A. Simonetti (1988). Food habits of Lama guanicoe in the Atacama Desert of northern Chile. Journal of Mammology 69: 198-201.

Ramírez, R. (1988). Morfología y biología de Bostryx conspersus (Sowerby) (Mollusca, Bulimulidae) en las lomas costeras del Perú Central. Revista Brasileira de Zoologia 5: 609-617.

Ramírez, R., S. Córdova, K. Caro y J. Duárez (2003). Response of a Land Snail Species (Bostyrx conspersus) in the Peruvian Central Coast lomas ecosystem to the 1982-1983 and 1997-1998 El Niño Events. En J. Haas y M. O. Dillon (eds.), El Niño in Peru: Biology and Culture over 10,000 Years, Fieldiana (Botany): 1-9. Chicago, Field Museum of Natural History.

Reitz, E. J. (2003). Resource Use through Time at Paloma Peru. Bulletin of the Florida Museum of Natural History 44:65-80.

Reus, M. L., C. M. Campos, S. M. Giannoni, S. G. Ortiz, C. A. Laspina, A. C. Navas y B. Peco (2009). Guanaco vs. donkey: what are they eating at Ischigualasto Park (San Juan)? Trabajo presentado en el $X$ International Mammalogical Congress, SAREM, Mendoza.

Richardson III, J. B. (1998). Looking in the Right Places: Pre-5,000 BP Maritime Adaptations in Peru and the Changing Environment. Revista de Arqueología Americana 15: 33-56.

Rizner, M., N. Vukosavljevi囚, N. y P. Miracle (2005). The paleoecological and paleodietary significance of edible land snails (Helix sp.) across the Pleistocene-Holocene transition on the eastern Adriatic Coast. En: S. McCartan, R. Schulting, G. Warren y P. Woodman (eds.), Mesolithic Horizons Papers Presented at the Seventh International Conference on the Mesolithic in Europe: 527-532. Oxford, Oxbow.

Rodbell, D. T., G. O. Seltzer, D. M. Anderson, M. B. Abbott, D. B. Enfield y J. H. Newman (1999). An 15,000-Year Record of El Niño-Driven Alluviation in Southwestern Ecuador. Science 283: 516-520.

Rostworowski, M. (1981). Recursos Naturales Renovables y Pesca, Siglos XVI y XVII. Lima, Instituto de Estudios Peruano.

Sandweiss, D. H. (2009). Early fishing and inland monuments: Challenging the maritime foundations of Andean civilization? En J. Marcus y P. W. Williams (eds.), Andean Civilization. A Tribute to Michael Moseley: 39-54. Monograph 63. Los Angeles, Cotsen Institute of Archaeology, University of California.

Sandweiss, D. H., C.F.T. Andrus, A. R. Kelley, K. A. Maasch, E. J. Reitz y P.B. Roscoe (2020). Archaeological climate proxies and the complexities of reconstructing Holocene El Niño in coastal Peru. PNAS 117: 8271-8279.

Sandweiss, D. H. y A. R. Kelley (2012). Archaeological Contributions to Climate Change Research: The Archaeological Record as a Paleoclimatic and Paleoenvironmental Archive. Annual Review of Anthropology 41: 371-391.

Sandweiss, D. H., J. B. Richardson III, E. J. Reitz, H. B. Rollins, K. A. Maasch (1996). Geoarchaeological evidence from Peru for a 5000 years BP onset of El Niño. Science 273: 1531-1533.

Shady, R. S. and C. Leyva, (eds.) (2003). La ciudad sagrada del Caral-Supe: los orígenes de la civilización andina y la formación del estado prístino en el antiguo Perú. Lima, Instituto Nacional de Cultura. 
DAVID G. BERESFORD-JONES Y OTROS - REVISITANDO LA HIPÓTESIS DE FREDERIC ENGEL: CÓMO LAS LOMAS ...

Simpson, B. B. (1989). "Krameriaceae”. Flora Neotropica 49: 1-108.

Stafford, D. (1939). On the Flora of Southern Peru. Proceedings of the Linnean Society of London 151: 172-181.

Strong, W. D. (1957). Paracas, Nazca, and Tiahuanacoid Cultural Relationships in South Coastal Peru. Memoirs of the Society for American Archaeology 13: 1-43.

Tripcevich, N., y D. A. Contreras (2011). Quarrying Evidence at the Quispisisa Obsidian Source, Ayacucho, Peru. Latin American Antiquity 22: 121-136.

Trujillo, D., y A. Delgado Rodríguez (2011). Aa from lomas formations. A new Orchidaceae record from the desert coast of Peru. Lankesteriana 11(1): 33-38. DOI 10.15517/lank.v11i1.18313.

Velarde, L. (2002-03). Frederic Engel y La Arqueología de La Costa Sur Del Perú. Société Suisse Des Américanistes Bulletin 66-67: 95-99.

Villagrán, C. y V. Castro (2003). Ciencia indígena de los Andes del norte de Chile. Santiago de Chile, Editorial Universitaria.

Vogt, B. (2011). Las Brujas, Bajo Río Grande (Perú). Jahresbericht 2010. Vol. 1. Deutsches Archäologisches Institut: 307-308.

Walter, H. (1973). Die Vegetation der Erde in öko-physiologischer Betrachtung: Die tropischen und subtropischen Zonen. Stuttgart. Fischer-Verlag, Stuttgart.

Waselkov, G. A. (1987). Shellfish Gathering and Shell Midden Archaeology. Advances in Archaeological Method and Theory 10: 93-210.

Weir, G. W. y J. P. Derring (1986). The Lomas of Paloma: Human-Environment Relations in a Central Peruvian Fog Oasis: Archaeobotany and Palynology, En R. Matos-M, S. A. Turpin y H. H. Eling (eds.), Andean Archaeology: Papers in Memory of Clifford Evans: 18-44. Los Angeles, Institute of Archaeology, University of California.

Wells, L. E. y J. S. Noller (1999). Holocene Coevolution of the Physical Landscape and Human Settlement in Northern Coastal Peru. Geoarchaeology 14: 755-789.

Whaley, O. Q., A. Orellana, E. Pérez, M. Tenorio, F. Quinteros, M. Mendoza y O. Pecho (2010). Plantas y Vegetación de Ica, Perú-Un recurso para su restauración y conservación. Royal Botanic Gardens, Kew.

Wheeler, J. C., D. H. Roque, D. Castillo, A. M. Porter, Y. I. Umire, Á. V. Anchea, A. R. Hawkins, R. R. Huayhua, L. M. Hernández y J. M. A. León (2011). Estudio de línea base, uso de hábitat y plan de manejo del guanaco informe final (enero - noviembre 2011). Lima, Instituto de Investigación y Desarrollo de Camélidos Sudamericanos CONOPA.

Willey, G. R. (1971). An Introduction to American Archaeology. Volume Two. Nueva Jersey, South America. Prentice-Hall.

Willis, K. J., y T. H. Van Andel (2004). Trees or no trees? The environments of central and eastern Europe during the Last Glaciation. Quaternary Science Reviews 23: 2369-2387.

Zeballos, H., L. Villegas, R. Gutiérrez y P. Jiménez (2000). Vertebrados de las lomas de Atiquipa y Mejía, sur del Perú. Revista Ecología Latinoamericana 7: 11-18.

Zeder, M. A. (2012). The Broad Spectrum Revolution at 40: Resource diversity, intensification, and an alternative to optimal foraging explanations. Journal of Anthropological Archaeology 31: 241-264. 
